\title{
Mitotic Motor KIFC1 Is an Organizer of Microtubules in the Axon
}

\author{
Hemalatha Muralidharan and Peter W. Baas \\ Drexel University College of Medicine, Department of Neurobiology and Anatomy, Philadelphia, Pennsylvania 19129
}

KIFC1 (also called HSET or kinesin-14a) is best known as a multifunctional motor protein essential for mitosis. The present studies are the first to explore KIFC1 in terminally postmitotic neurons. Using RNA interference to partially deplete KIFC1 from rat neurons (from animals of either gender) in culture, pharmacologic agents that inhibit KIFC1, and expression of mutant KIFC1 constructs, we demonstrate critical roles for KIFC1 in regulating axonal growth and retraction as well as growth cone morphology. Experimental manipulations of KIFC1 elicit morphological changes in the axon as well as changes in the organization, distribution, and polarity orientation of its microtubules. Together, the results indicate a mechanism by which KIFC1 binds to microtubules in the axon and slides them into alignment in an ATP-dependent fashion and then cross-links them in an ATP-independent fashion to oppose their subsequent sliding by other motors.

Key words: axon; growth cone; KIFC1; kinesin-14; microtubule; neuronal polarity

\section{Significance Statement}

Here, we establish that KIFC1, a molecular motor well characterized in mitosis, is robustly expressed in neurons, where it has profound influence on the organization of microtubules in a number of different functional contexts. KIFC1 may help answer long-standing questions in cellular neuroscience such as, mechanistically, how growth cones stall and how axonal microtubules resist forces that would otherwise cause the axon to retract. Knowledge about KIFC1 may help researchers to devise strategies for treating disorders of the nervous system involving axonal retraction given that KIFC1 is expressed in adult neurons as well as developing neurons.

\section{Introduction}

Several proteins contribute to the organization of microtubules in the axon. We have emphasized the importance of molecular motor proteins, not only in transporting microtubules within the axon (Baas and Ahmad, 2001; Baas, 2002; He et al., 2005; Ahmad et al., 2006; Baas et al., 2006; Myers and Baas, 2007; Liu et al., 2010; Lin et al., 2012; Rao et al., 2017), but also in imposing order on the microtubules (Rao and Baas, 2018) and orchestrating a variety of force-related mechanisms underlying events in the life of the neuron such as growth cone turning (Myers et al., 2006; Nadar et al., 2008, 2012; Liu et al., 2010) and

\footnotetext{
Received Dec. 11, 2018; revised Jan. 30, 2019; accepted Feb. 18, 2019.

Author contributions: H.M. and P.W.B. designed research; H.M. performed research; H.M. and P.W.B. analyzed data; H.M. and P.W.B. wrote the first draft of the paper; H.M. and P.W.B. edited the paper; H.M. and P.W.B. wrote the paper.

This work was supported by the National Institute of Neurological Disorders and Stroke (Grant R01 NS28785) to P.W.B. We thank Claire Walzack for providing us with the HSET constructs, Vladimir Zhukarev for assistance with microscopy, Timothy Mitchison for providing FCPT, and Liang Qiang, Wenqian Yu, and Kiran Madugula for helpful discussions and technical advice.

The authors declare no competing financial interests.

Correspondence should be addressed to PeterW. Baas at pwb22@drexel.edu.

https://doi.org/10.1523/JNEUROSCI.3099-18.2019

Copyright $\odot 2019$ the authors
}

neuronal migration (Buster et al., 2003; Falnikar et al., 2011; Rao et al., 2016).

Cytoplasmic dynein is the chief molecular motor for transporting neuronal microtubules and imposing forces on them (Ahmad et al., 1998, 2006; He et al., 2005; Rao et al., 2017), but a variety of other motors contribute as well, most of which are kinesins best known for their roles in mitosis. Some of these motors act as energy-burning brakes (Myers and Baas, 2007; Liu et al., 2010; Del Castillo et al., 2015) that impede or slow the motion induced by other motor proteins. The most robust contributions of the mitotic motors studied to date in neurons occur during development, with the levels of the motors waning as the nervous system matures to adulthood (Ferhat et al., 1998a,b; Myers and Baas, 2007; Liu et al., 2010; Lin et al., 2011, 2012). KIFC1 (also called HSET or kinesin-14a) is the most highly expressed force-generating mitotic kinesin in neurons and, unlike the others, continues to be highly expressed throughout the life of the nervous system (Silverman et al., 2010). However, until now, KIFC1 had never been studied in the nervous system beyond documentation of its high expression.

Kinesin-14 family members are unique among kinesins in that their motor domain is on the $\mathrm{C}$ terminus rather than $\mathrm{N}$ terminus 
A

Developmental expression

Hippocampus

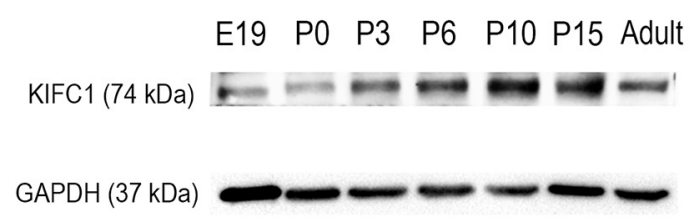

C

Knockdown efficiency

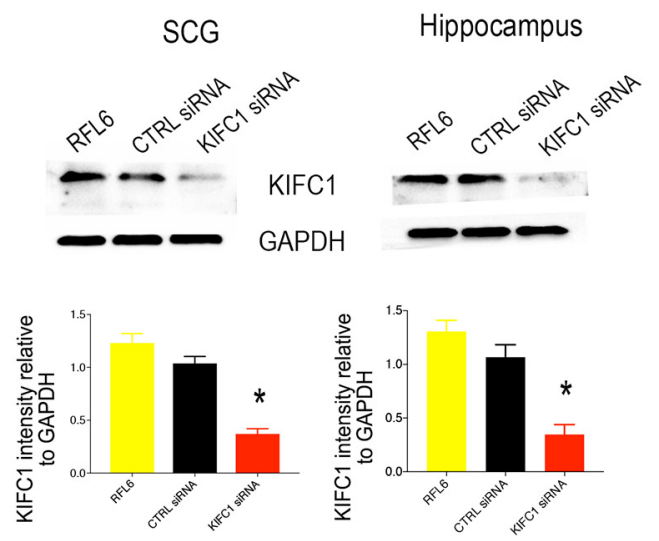

B

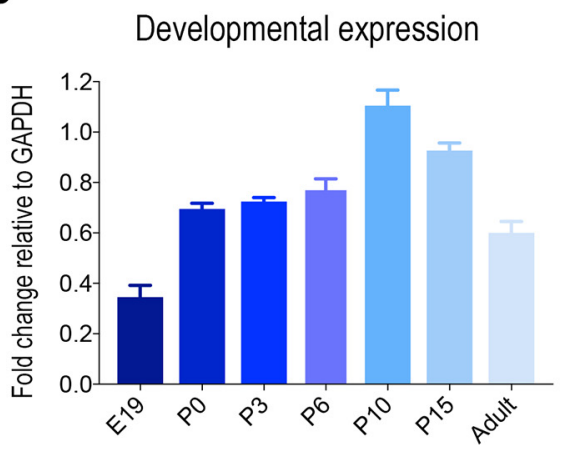

D

KIFC1 fluorescence intensity

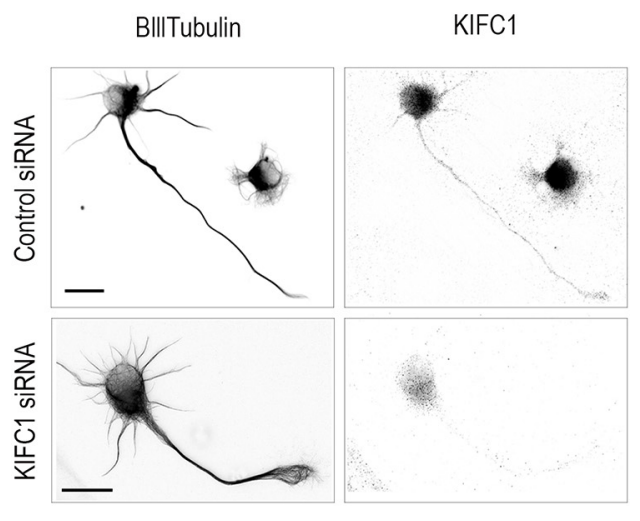

Figure 1. KIFC1 is expressed in neurons. $\boldsymbol{A}$, Western blot representing KIFC1 expression in rat hippocampus tissue lysates at different developmental ages. $\boldsymbol{B}$, Bar graph representing KIFC1 expression in rat hippocampus tissue lysates at different developmental ages. C, Quantification and analysis by Western blot of KIFC1 depletion efficiency after siRNA treatment of rat fetal hippocampal or rat newborn sympathetic (SCG) cultures. Quantifications are an average of at least three independent experiments $\left({ }^{*} p<0.05\right.$ ). D, Immunofluorescence images (displayed as inverted images for better contrast) of cultured rat hippocampal neurons showing depletion of KIFC1. The neuron was immunostained for $\beta \mathrm{III}-$ tubulin (left) and KIFC1 (right). Data are represented as mean \pm SEM. All statistical test comparisons were done against the control group with the KIFC1 siRNA group. ANOVA test and further multiple comparisons were made using Bonferroni test. Scale bar in $\boldsymbol{D}, 20 \mu \mathrm{m}$ (top) and $10 \mu \mathrm{m}$ (bottom).

and that they move toward minus ends of microtubules rather than plus ends (She and Yang, 2017). KIFC1 is the kinesin-14 that participates in mitosis (Cai et al., 2009; Hepperla et al., 2014), regulating the sliding of microtubules (Walczak et al., 1998; Mountain et al., 1999; Simeonov et al., 2009; Weaver et al., 2015; Braun et al., 2017), whereas KIFC2 is the kinesin-14 that transports membranous vesicles (Hanlon et al., 1997; Saito et al., 1997; Yang et al., 2001). In studies on mitosis and in vitro, KIFC1 has been shown to slide microtubules relative to one another (Braun et al., 2009; Fink et al., 2009; She and Yang, 2017), including drawing adjacent microtubules together (Molodtsov et al., 2016) and also to cross-link microtubules in an ATP-independent fashion that impedes their sliding (Mountain et al., 1999; Sharp et al., 2000; Cai et al., 2009; She and Yang, 2017).

Our first thought about KIFC1 in neurons was that it would serve as a backup to the functions of cytoplasmic dynein related to microtubule sliding, namely transporting short microtubules in a polarity-sorting manner in the axon (Rao et al., 2017) and also enabling long microtubules to resist the compressive and retrograde forces of actomyosin in the axon (Ahmad et al., 2000; Myers et al., 2006; Grabham et al., 2007). However, once we began studying KIFC1 in the neuron, it became clear that, whereas some of the effects of its inhibition are similar to those of inhibiting dynein, others are quite different, reminiscent of KIFC1's specialized sliding and cross-linking properties in the mitotic spindle.
Here, we document for the first time that inhibition or depletion of KIFC1 from cultured vertebrate neurons has profound effects on axonal growth and retraction, as well as microtubule polarity orientation and growth cone morphology. From there, we focus on the mechanisms underlying these effects.

\section{Materials and Methods}

Neuronal culture and siRNA-based depletion of KIFC1. All animals were used according to National Institutes of Health and Drexel University institutional animal care and use committee guidelines. Hippocampi were dissected from Timed-Pregnant (TP18 to TP19) Sprague Dawley rat fetuses of either sex, and then cultured as previously described (Qiang et al., 2006). Superior cervical ganglia (SCG) were acquired from postnatal day 0 (P0) to P3 Sprague Dawley rat pups of either sex and cultured as described previously (Myers and Baas, 2007; Liu et al., 2010). Control scrambled siRNA (Sigma-Aldrich, SIC001) or KIFC1 siRNA (SigmaAldrich, SASI_Rn01_00080300, SASI_Rn01_00080301, SASI_Rn01_ 00080302, SASI_Rn01_00080304) was introduced into neurons by nucleofection (Nucleofector $2 \mathrm{~b}$, Lonza) before plating. The cells were cultured for 36 or $60 \mathrm{~h}$ (to allow for KIFC1 to be partially depleted) on 35 -mm-diameter culture dishes coated with $1 \mathrm{mg} / \mathrm{ml}$ poly-L-lysine (Sigma-Aldrich, P2636-25MG) or $0.1 \mathrm{mg} / \mathrm{ml}$ poly-D-lysine (SigmaAldrich, P0899-50MG) and then replated on glass-bottomed dishes (Cellvis, \#D35-14-1.5-N) coated with $1 \mathrm{mg} / \mathrm{ml}$ poly-L-lysine or 0.1 $\mathrm{mg} / \mathrm{ml}$ poly-D-lysine. SCGs were replated on laminin (Invitrogen\# 23017-015) (3 h before plating)/poly-D-lysine after $60 \mathrm{~h}$ of siRNA treat- 
A

Cell viability

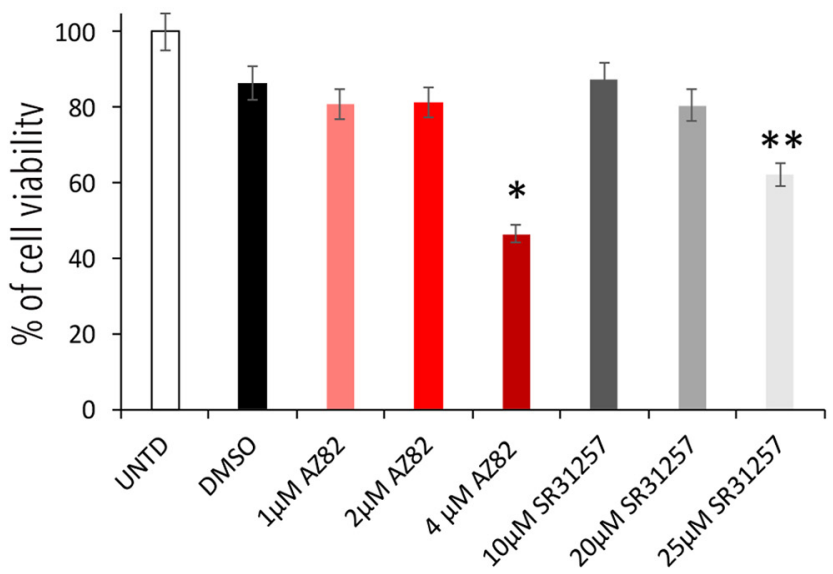

B

Drug concentrations

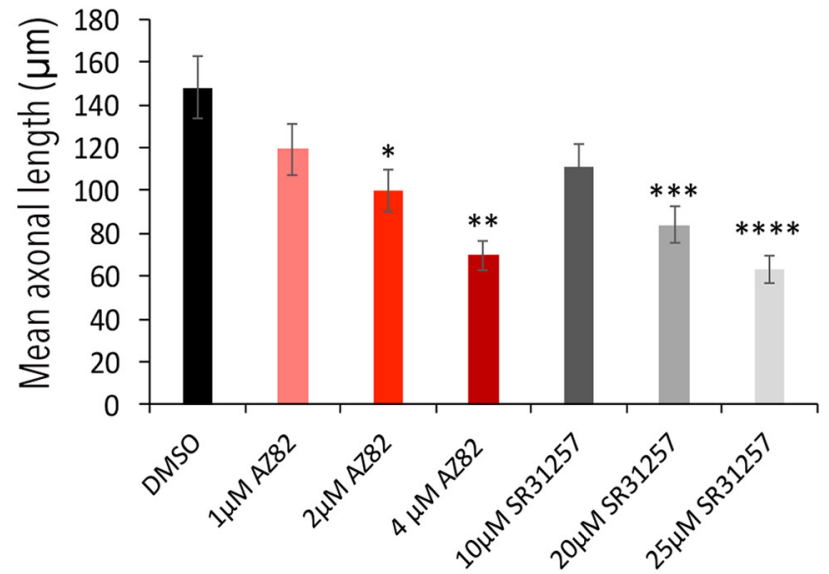

C

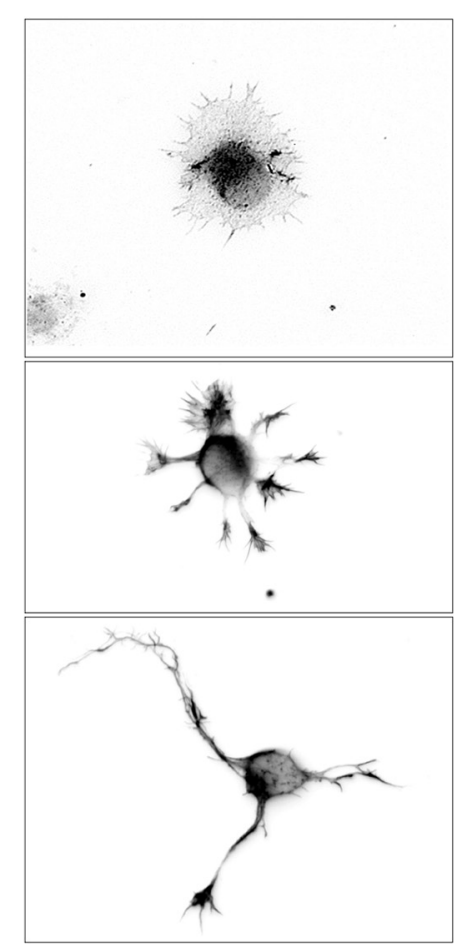

D

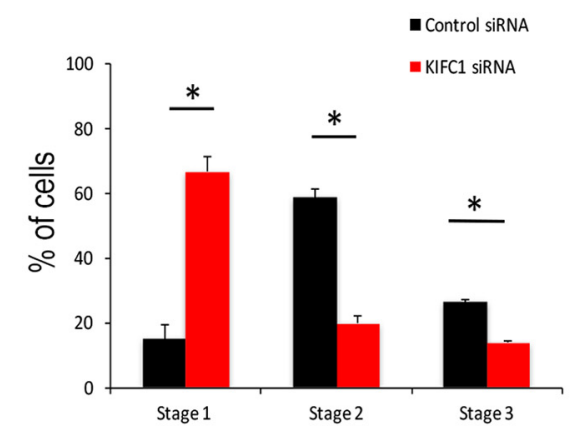

E Cell polarization after 24 hours

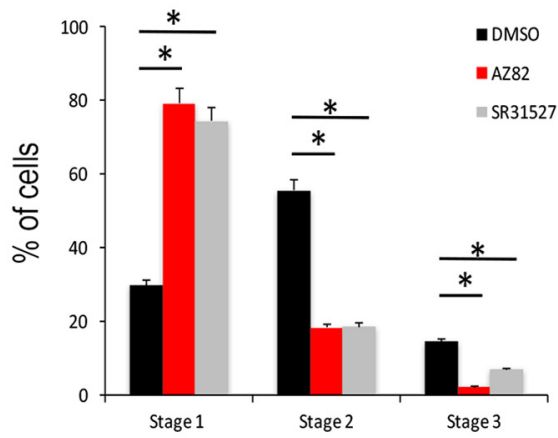

D'

Cell polarization after 48 hours

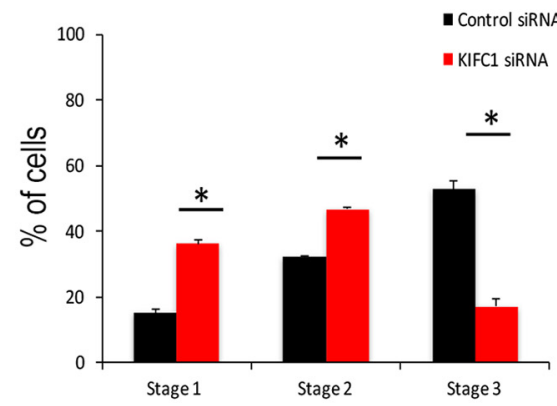

Cell polarization after 48 hours

Figure 2. KIFC1 depletion delays cell polarization. $A$, Bar graph representing percentage of neuronal cell viability (as determined with MTT assay) after various concentrations of the two KIFC1 drugs (SR31527 and AZ82). ${ }^{*} p<0.05$ comparing DMSO vs $4 \mu$ M AZ82, ${ }^{* *} p<0.05$ comparing DMSO vs $25 \mu$ M SR31257. B, Bar graph representing mean axonal length of neurons after various treatments with the two different KIFC1 drugs. ${ }^{*} p<0.05$ comparing DMSO vs $2 \mu$ M AZ82, ${ }^{* *} p<0.05$ comparing DMSO vs $4 \mu$ M AZ82, ${ }^{* * *} p<0.05$ comparing DMSO vs $20 \mu M$ SR31257, ${ }^{* * * *} p<$ 0.05 comparing DMSO vs $25 \mu$ M SR31257. C, Different stages of the development of hippocampal neurons in culture as shown by immunofluorescence images of cultures stained for $\beta$ III-tubulin displayed as inverted images for better contrast. $\boldsymbol{D}$, Average percentage of neurons at each stage of development after $24 \mathrm{~h}(\boldsymbol{D})$ and $48 \mathrm{~h}\left(\boldsymbol{D}^{\prime}\right)$ of replating in control and KIFC 1 depletion groups. ${ }^{*} p<$ 0.05. $\boldsymbol{E}$, Average percentage of hippocampal neurons at each stage in culture after $24 \mathrm{~h}(\boldsymbol{E})$ and $48 \mathrm{~h}\left(\boldsymbol{E}^{\prime}\right)$ of treatment in untreated and DMSO-, AZ82-, and SR31527-treated groups. ${ }^{*} p<0.05$. Scale bars, $10 \mu \mathrm{m}$ (stage 1 and 2 neurons), $15 \mu \mathrm{m}$ (stage 3 neuron).

ment. For experiments involving hippocampal neurons, the cells were replated on poly-L-lysine-coated glass-bottomed dishes after $36 \mathrm{~h}$ of siRNA treatment.

KIFC1 constructs. Full-length non-mutated human KIFC1 (termed HSET) was obtained as a GFP-fusion from Dr. Claire Walzcak. A mutant form (HSET-N593K), which uncouples microtubule binding and microtubule-stimulated ATPase activity (Cai et al., 2009), was prepared and provided by Dr. Claire Walczak, on the basis of previous work on the
Drosophila kinesin-14 NCD (Song and Endow, 1996). This mutant, which we refer to as the cross-linking-only mutant, has the ability to cross-link microtubules but cannot slide or transport them. Dr. Claire Walczak also provided us with KIFC1 truncated mutant (termed HSETMotor-Stalk, consisting of 145-673 aa), which does not contain the tail domain. KIFC1 rigor mutant was prepared in our laboratory on the basis of such a mutant previously developed for NCD (Oladipo et al., 2007); correspondingly, the amino acid mutation (HSET-Rigor-T417N) was 

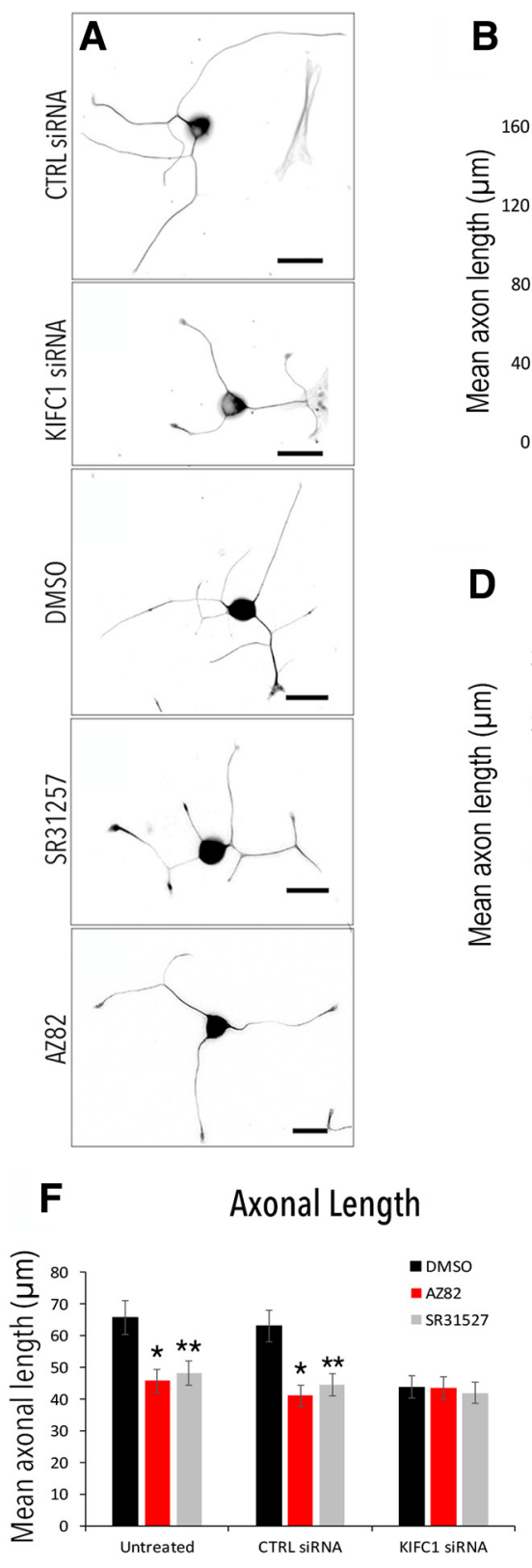

B

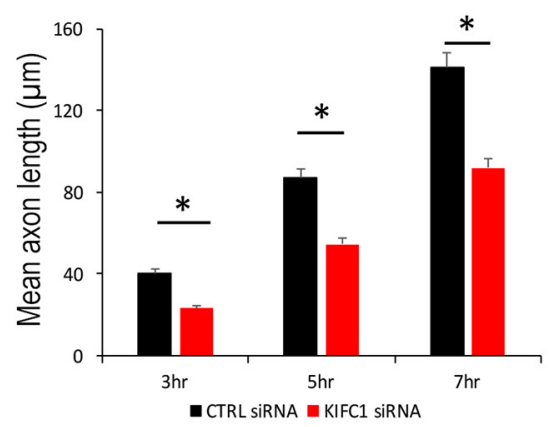

D Primary axon

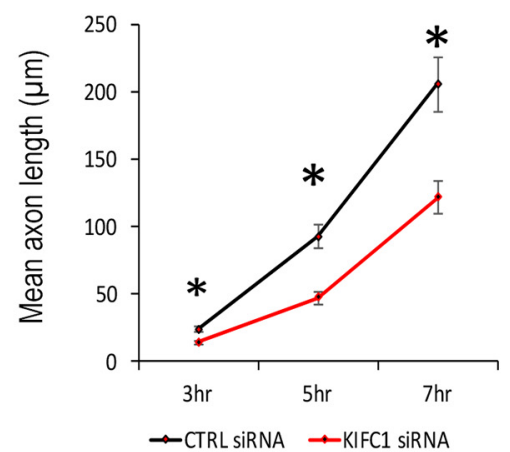

G

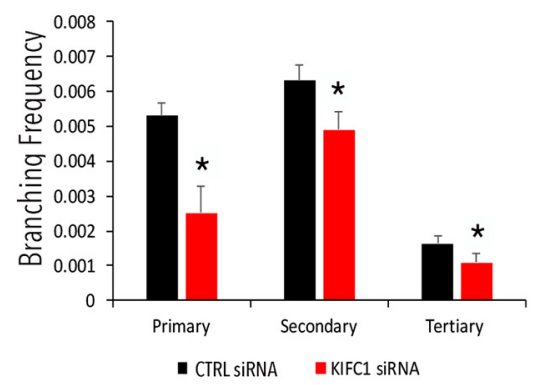

C Mean axon length

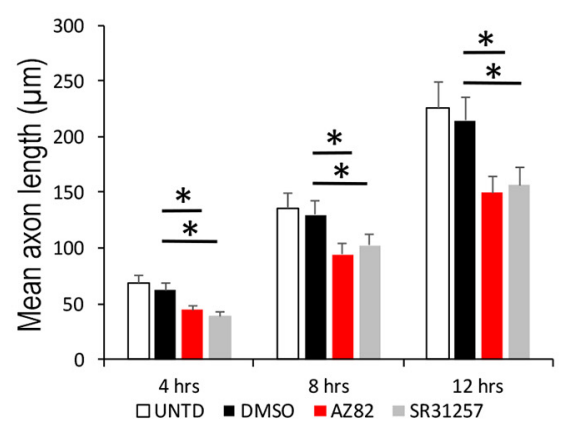

$\mathbf{E}$

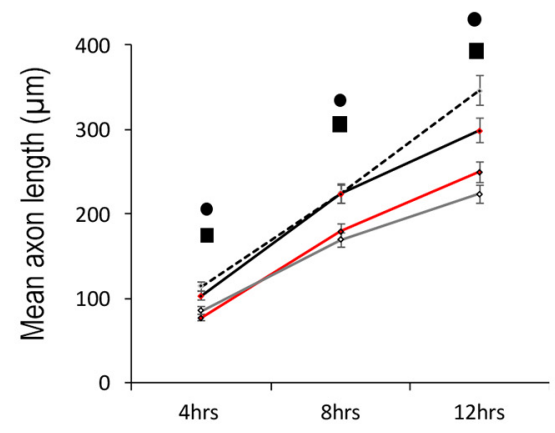

-.-. UNTD - -DMSO - - AZ82 —-SR31257
H Branch formation

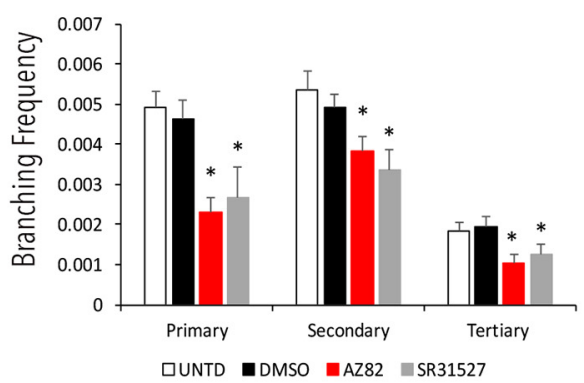

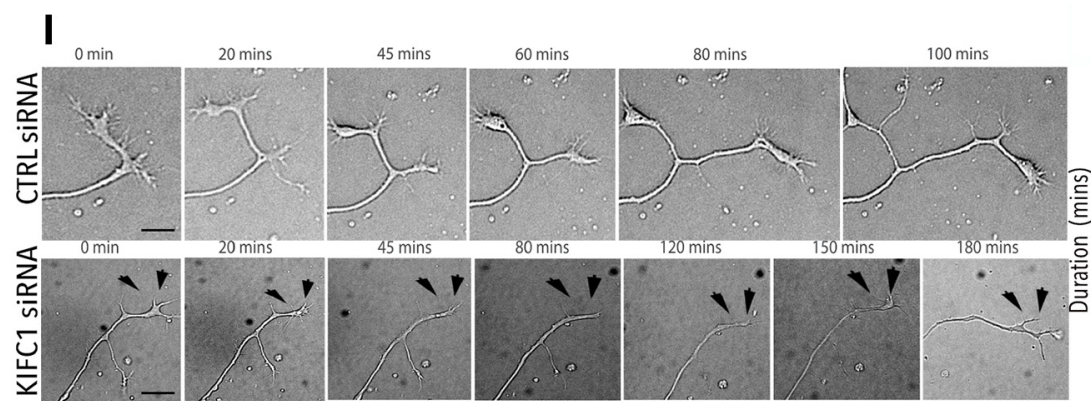

J Stall Duration

K Axonal growth
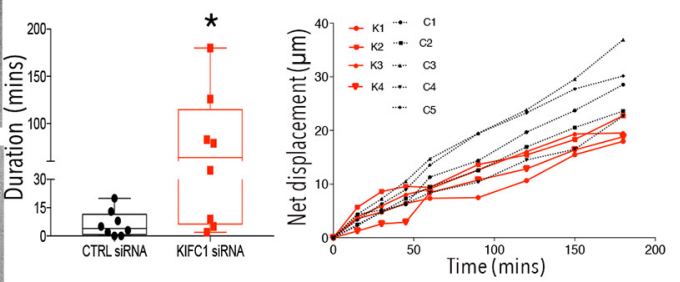

Figure 3. KIFC1 depletion affects axon formation. $A$, Representative immunofluorescence images of sympathetic (SCG) neurons that had been transfected with control siRNA or KIFC1 siRNA and treated with DMSO, SR31527, or AZ82 and stained for $\beta$ III-tubulin displayed as inverted images for better contrast. $\boldsymbol{B}$, Bar graphs representing quantification of mean axonal length of control or KIFC1-depleted sympathetic (SCG) neurons at 3, 5 , and $7 \mathrm{~h}$ after replating. ${ }^{*} p<0.05 ; n=30$ per group. C, Bar graphs representing quantification of mean axonal length of untreated and DMSO-, AZ82-, or SR31527-treated sympathetic (SCG) neurons at 4,8 , and $12 \mathrm{~h} .{ }^{*} p<0.05 ; n=25$ per group. $\boldsymbol{D}$, Line graph representing mean primary branch axonal length after 3,5 , and $7 \mathrm{~h}$ after replating sympathetic (SCG) neurons subjected to control or KIFC1 siRNA treatments. ${ }^{*} p<0.05 ; n=30$ per group. $E$, Line graph representing mean primary branch axonal length of untreated and DMSO-, AZ82-, or SR31527-treated sympathetic (SCG) neurons at 4, 8, and $12 \mathrm{~h}$. $\square p<0.05$ comparing DMSO versus AZ82, $p<0.05$ comparing DMSO versus SR31527; $n=25$ per group. $\boldsymbol{F}$, Bar graph representing mean axonal length of untreated, control siRNA, or KIFC1 siRNA neurons treated with DMSO, SR31527, or AZ82. DMSO is the vehicle into which the (Figure legend continues.) 
introduced in the HSET plasmid (provided by Dr. Claire Walzack) using the Quikchange site-directed mutagenesis system (Stratagene). The siRNA sequences chosen to target rat KIFC1 do not affect human KIFC1/ HSET, permitting us to use human constructs for rescue experiments (either wild-type or mutants).

Drugs. For inhibition of KIFC1, two different drugs were used, AZ82 (AstraZeneca) and SR31527 (Vitascreen), each with different properties (Wu et al., 2013; Yang et al., 2014; Zhang et al., 2016; Park et al., 2017). For one set of studies, vinblastine sulfate (Sigma-Aldrich) was used to suppress microtubule dynamics (Ahmad et al., 1998) and FCPT was used to suppress microtubule transport (Rao et al., 2016, 2017). (2-(1-(4fluorophenyl)cyclopropyl)-4-(pyridin-4-yl)thiazole); FCPT was provided as a gift from Dr. Timothy Mitchison.

Immunofluorescence. For almost all experiments (unless otherwise stated), cultures were co-extracted and fixed for $12 \mathrm{~min}$ in a solution containing $4 \%$ paraformaldehyde, $1 \times$ PHEM buffer (PIPES, HEPES, EDTA, $\mathrm{MgCl}_{2}$ ), $0.2 \%$ glutaraldehyde, and $0.1 \%$ Triton X-100. Glutaraldehyde was then quenched by treatment for $15 \mathrm{~min}$ each with $3 \mathrm{mg} / \mathrm{ml}$ sodium borohydride. Cultures were then blocked for $1 \mathrm{~h}$ with normal goat serum (Jackson ImmunoResearch, \#005-000-121), followed by incubation with primary antibodies overnight at $4^{\circ} \mathrm{C}$ and then secondary antibodies for $2 \mathrm{~h}$ at room temperature.

Primary antibodies were as follows: rabbit anti- $\beta$ III-tubulin (1:1500; BioLegend, \#802001), mouse anti- $\beta$ III-tubulin (1:1500; BioLegend, \#801202), rabbit anti-KIFC1 (1:800; Proteintech, \#20790-1-AP-Western blot); rabbit anti-KIFC1 (1:40; Novus Biological, \#NB100-40844); and synaptophysin, rabbit anti-MAP2 (1:1200; AB5622). Appropriate secondary antibodies were purchased from Jackson ImmunoResearch and used accordingly. Alexa Fluor 488 phalloidin (1:40; Molecular Probes, \#A12379) or rhodamine phalloidin (1:40; Invitrogen, \#R418) was used for actin staining.

Western blotting. All Western blots were replicated three times. Hippocampi from different-aged rats were dissected and flash frozen using cooled 2-methyl butane. Protein lysates were obtained from cultures or tissues using RIPA buffer (Thermo Fisher Scientific, 89901) supplemented with protease inhibitor mixture (Thermo Fisher Scientific, 78430) and Phosphatase Inhibitor Cocktail 3 (Sigma-Aldrich, \#P0044$1 \mathrm{ML}$ ). Total protein concentration in the lysates was measured using the BCA protein assay (Thermo Fisher Scientific, 23227). Protein samples of $35-40 \mu \mathrm{g}$ were loaded per lane of $4-15 \%$ gradient SDS-PAGE gels (BioRad, \#456-1084), after which transfer was conducted onto PVDF membranes (Bio-Rad, \#162-0177) at $4^{\circ} \mathrm{C}$ for $16-18 \mathrm{~h}$ at $30 \mathrm{~V}$. Membranes were then blocked for $1 \mathrm{~h}$ in 5\% fat-free milk (Lab Scientific, \#M0841) in $1 \times$ TBS-T (10× TBS; Bio-Rad, \#170-6435). Rabbit anti-KIFC1 (Proteintech, \#20790-1-AP), rabbit anti-cofilin (Abcam, \#42824) or mouse antiGAPDH (1:5000; Abcam, \#ab8245) were the primary antibodies. The latter two served as loading controls. Appropriate secondary antibodies were peroxidase conjugated. Blots were visualized using a combination of the Super Signal West Pico kit (Thermo Fisher Scientific, 34580) and the Super Signal West Femto kit (Thermo Fisher Scientific, \#34094). The

\section{$\leftarrow$}

(Figure legend continued.) drugs are dissolved and serves as the control. For untreated group, ${ }^{*} p<0.05$ comparing DMSO vs AZ82 and ** $p<0.05$ comparing DMSO vs SR31257. Similarly, for control siRNA group, ${ }^{*} p<0.05$ comparing DMSO vs AZ82 and ${ }^{* *} p<0.05$ comparing DMSO vs $S R 31257$ respectively. $G$, Bar graph representing branching frequency of primary, secondary, and tertiary branches of control and KIFC1-depleted neurons. ${ }^{*} p<0.05, n=30$ per group. $\boldsymbol{H}$, Bar graph representing branching frequency of primary, secondary, and tertiary branches of untreated and DMSO-, AZ82-, or SR31527-treated neurons. ${ }^{*} p<0.05, n=25$ per group. $I$, Representative phase-contrast images of the growing tip of the axon comparing control or KIFC1 siRNA groups over time. J, Bar graph representing stall duration comparison between control and KIFC 1 siRNA neurons. ${ }^{*} p<0.05, n=8$ per group. $\boldsymbol{K}$, Line graph representing the net displacement of the growing tip of the axon comparing the control and KIFC1 siRNA groups. Data are represented as mean \pm SEM. Unless otherwise specified, all statistical test comparisons were done against control siRNA group with KIFC1 siRNA group in the case of depletion experiments. For drug studies, comparisons were made against DMSO versus untreated or AZ82 or SR31527 treatments using ANOVA test. Further multiple comparisons were made using Bonferroni test. Scale bars: $A, 50 \mu \mathrm{m} ; I, 15 \mu \mathrm{m}$. intensity of the bands on the blots was measured using Image Lab (Bio-Rad).

Morphological studies. For cell polarization studies, hippocampal cells were cultured for $36 \mathrm{~h}$ after transfection with either control or KIFC1 siRNA and then replated on glass-bottomed dishes. After 24 or $48 \mathrm{~h}$, the replated cells were fixed and immunostained for further analysis. For other morphological studies including axonal length measurements and branching frequencies, sympathetic (SCG) neurons were cultured and replated on laminin-coated dishes after the siRNA regimen. In some studies, KIFC1 drugs or their DMSO vehicle were added at the time of plating. All morphological studies on sympathetic (SCG) neurons were conducted on cells grown on laminin. For axonal length and branching studies, neurons were analyzed after 3,5 , and $7 \mathrm{~h}$ in the case of the siRNA regimen and 4, 8, and $12 \mathrm{~h}$ in the case of the drug regimen. All of the data on axons were acquired from neurons in stage 3 of development, wherein the axon is defined as the longest process. Staining for $\beta$ III-tubulin (which is neuron specific) further confirmed neuronal identity, such that the longest process of a confirmed neuron in these cultures was unquestionably the axon. Axonal lengths were measured using ImageJ software and mean values were quantified. Analysis of branching frequency was performed by counting the total number of branches per neuron and dividing these values by total axonal length for individual neurons. Growth cone area and perimeter were traced using ImageJ software.

EB3-GFP assay. EB3-GFP plasmid was transfected into the neurons before replating. Live-cell imaging was conducted on an environmentally-controlled microscope stage, with images acquired at $1 \mathrm{~s}$ intervals for a total of 150 frames using the Zeiss $100 \times / 1.46$ Plan-Achromat objective. For rescue experiment and mutant experiments with GFPtagged KIFC1 constructs, an mCherry-EB3 plasmid was used. Images were later exported as multidimensional TIFF files. EB3 comets from these raw unprocessed images were tracked automatically using plusTipTracker software in MATLAB. To measure lifetime and comet rate of EB3 comets, the following parameters were set in the program: search radius range, 5-12 pixels. Comet speed was defined as the average velocity taken from multiple frames. To visualize comet tracks in individual growth cones and axons, the plusTipSeeTracks function was used. For statistical analysis, the plusTipGroupAnalysis function was used to compare the dynamic parameters among groups. Microtubule dynamics parameters were compiled from multiple individual experiments.

Full-width at half-maximum (FWHM). To measure the spread of a particular intensity along the length of the axon at a definitive period or over time we calculated the FWHM of the particular intensity profile. To perform automated measurements, a reference image that consisted of a line of pixels running along the axon segment was produced by thresholding and binarizing the images before using a plugin from ImageJ. The FWHM was measured automatically along the axon guided by the reference image. For every position, intensity profiles from two parallel lines were averaged to reduce noise and were calculated using MS excel.

Nitric oxide experiments on axonal retraction. The nitric oxide donor NOC-7 (Calbiochem) was prepared and used as described previously (Baas and Ahmad, 2001; He et al., 2002; Myers and Baas, 2007) at a working concentration of $0.3 \mu \mathrm{m}$. After $60 \mathrm{~h}$ of siRNA treatment, neurons were replated onto poly-D-lysine $(0.1 \mathrm{mg} / \mathrm{ml})$-coated and laminintreated $(25 \mu \mathrm{g} / \mathrm{ml})$ glass-bottomed culture dishes. By $72 \mathrm{~h}$, the neurons had generated extensive axons. Differential interference contrast (DIC) images of axons were recorded from 0 to $30 \mathrm{~min}$ for every minute after the addition of NOC-7. Growth cone displacement was measured using ImageJ. Raw data were processed and graphs were produced using Excel (Microsoft).

Microtubule transport/sliding assays. The assay for observation of short microtubules moving rapidly in the axon was performed as described previously (Myers and Baas, 2007; Rao et al., 2017). Sympathetic (SCG) neurons were transfected to express Td-Eos tubulin before plating, with imaging conducted $14 \mathrm{~h}$ after plating. Laminin was added to the medium to promote axonal outgrowth during the final $3 \mathrm{~h}$ before imaging. AZ82 was added just before imaging. For siRNA and mutant experiments, neurons were cotransfected with appropriate siRNA and KIFC1 (mutant or wild-type) plasmid, along with Td-Eos tubulin plasmid, and then plated. Imaging was conducted $36 \mathrm{~h}$ after transfection. All axons chosen 
A

Polar histogram plots of growth directions of the entire length of the axon.
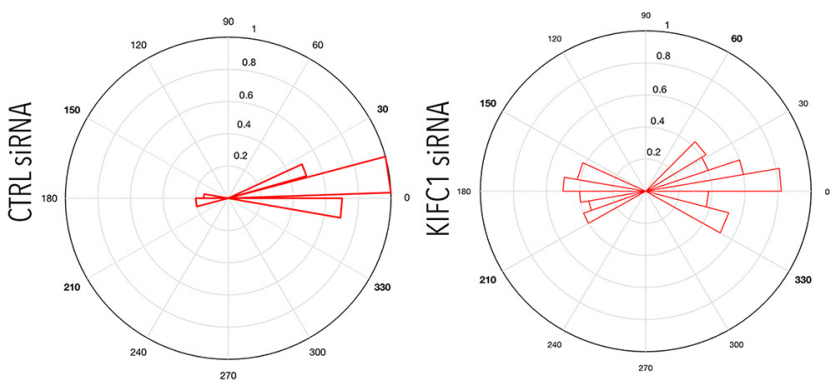

D

C

Entire length of the axon

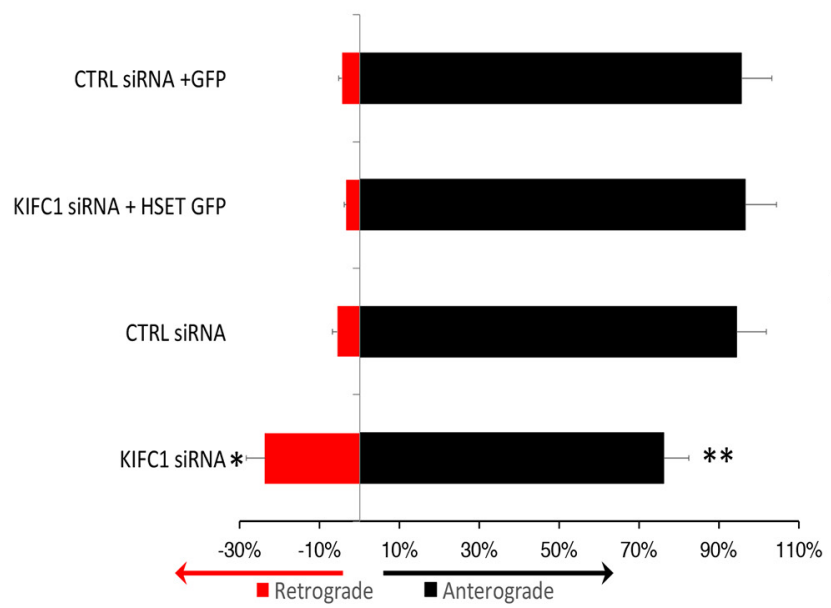

$\mathbf{F}$

EB3 comet speed

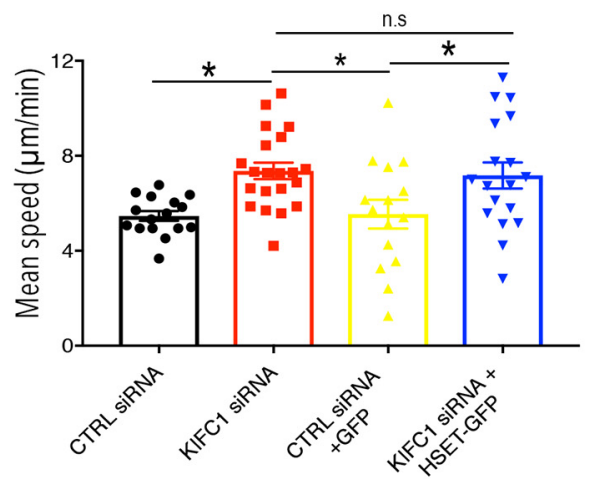

B
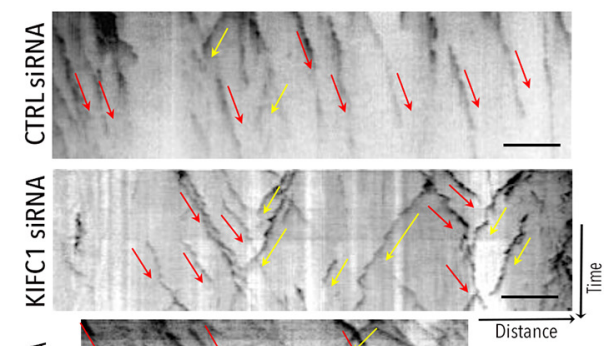

这造

它

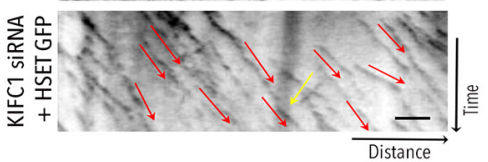

Distal third of the axon length

KIFC1 SiRNA

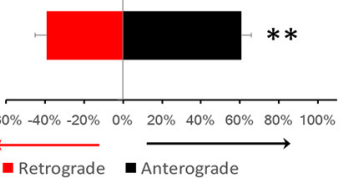

E

\section{Proximal two-thirds of the axon length}

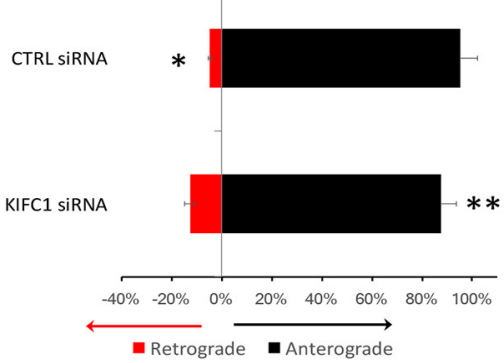

G

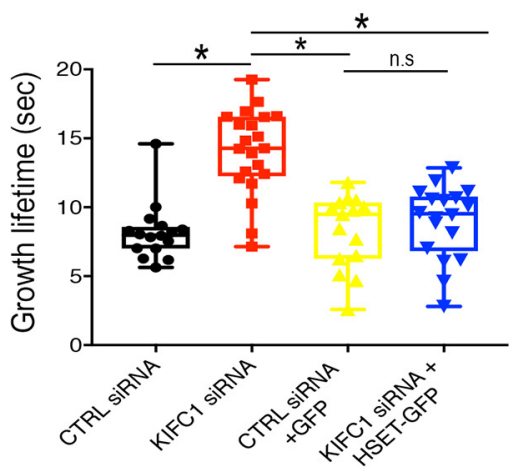

Figure 4. KIFC1 depletion induces microtubule polarity flaws in the axon. $A$, Radial polar histogram plots of comet growth direction of the entire axonal length from control siRNA (left) hippocampal neurons and KIFC1 siRNA hippocampal neurons (right). Control siRNA, $n=16$; KIFC1 siRNA, $n=21$. B, Representative kymographs from control, KIFC1-depleted neurons, control siRNA + GFP or KIFC1 siRNA + HSET-GFP, showing EB3-GFP movement. C, Quantification of EB3-GFP comet movement in the entire axonal length, in the distal axon (20\% of entire axonal length) (D), and in the remaining $80 \%$ of the axon $(\boldsymbol{E}) .{ }^{*} p<0.05$ for the comparison of anterograde EB3 movement; ${ }^{* *} p<0.05$ for the comparison of retrograde EB3 movement; control siRNA, $n=16$; KIFC1 siRNA, $n=21$; control siRNA + GFP, $n=15$; KIFC1 siRNA + HSET-GFP, $n=18$. $\boldsymbol{F}$, Bar graph representing mean comet speed from the entire length of the axons of control, KIFC1-depleted hippocampal neurons, control siRNA + GFP and KIFC1-depleted hippocampal neurons expressing HSET + GFP. ${ }^{*} p<0.05$; control siRNA, $n=16 ; \mathrm{KIFC}$ siRNA, $n=21$; control siRNA + GFP, $n=15$; and KIFC1 siRNA + HSET-GFP, $n=18$. G, Box plots represent EB3 comet growth lifetime of the entire length of the axons of control, KIFC1-depleted hippocampal neurons, control siRNA + GFP and KIFC1-depleted hippocampal neurons expressing HSET + GFP. Control siRNA, $n=16 ; \mathrm{KIFC} 1$ siRNA, $n=21$; control siRNA + GFP, $n=15 ; \mathrm{KIFC1} \mathrm{siRNA} \mathrm{+} \mathrm{HSET-GFP,} n=18$. (Figure legend continues.) 
for imaging were at least $100-150 \mu \mathrm{m}$ in length. A $30 \mu \mathrm{m}$ bleached/ photoconverted zone was created in the green channel using the Andor Mosaic system and imaging was conducted only in the green channel. Images were taken every $0.5 \mathrm{~s}$ for a total of 150 frames at $250 \mathrm{~ms}$ exposure using a Zeiss AxioObserver Z1 equipped with a $100 \times$ Plan-Apochromat objective (1.46 numerical aperture). Transport analysis included all microtubules observed to move through the bleached/photoconverted zone in the green channel during imaging. Transport frequencies were calculated by dividing the total number of movements by the total imaging time for each individual movie.

For observation of longer microtubules undergoing episodes of sliding in the axon, we used a modification of the procedure previously used in our recent study on migratory neurons (Rao et al., 2016). All mutant plasmids were cotransfected into dissociated cells together with the siRNA and Td-Eos tubulin plasmid and cells were then plated on glassbottomed dishes for $30 \mathrm{~h}$, after which laminin (1:500) was added to enhance axonal growth during the last $3 \mathrm{~h}$ before imaging. All axons chosen for imaging were at least $\sim 150 \mu \mathrm{m}$ in length. A bleached/photoconverted zone $\sim 30 \mu \mathrm{m}$ was made in the green channel in the distal third of the axon. Images were captured every $30 \mathrm{~s}$ for $\sim 10 \mathrm{~min}$ in the red channel using a Zeiss AxioObserver Z1 equipped with a $100 \times$ Plan Apochromat objective (1.46 numerical aperture) or using the Leica True Confocal System SP8. The Td-Eos in the bleached zone is "photoconverted" so that it fluoresces in the red channel. We assayed the decay over time of the red fluorescence as a readout of the sliding of long microtubules out of the bleached/photoconverted zone into the unbleached/ non-photoconverted zone. We also assayed the appearance of red fluorescence in the regions (unbleached/non-photoconverted zone) of the axon flanking the bleached/photoconverted zone as further evidence of the sliding of long microtubules.

Mitochondrial transport assay. To test specificity of the effects of KIFC1 on microtubule transport/sliding and organization, as opposed to potential effects on organelle transport, we assayed mitochondrial transport, as we did in our previous studies on kinesin-5 in neurons (Myers and Baas, 2007). Neurons were incubated for $30 \mathrm{~min}$ using medium Mito-Tracker $\mathrm{CMH}_{2}$ TMRos Orange (Molecular Probes, \#M7511). After incubation, dishes were washed twice with serum-free medium and then imaged with L-15 medium in the culture. Cells were imaged using a Zeiss AxioObserver Z1 equipped with a $100 \times$ Plan Apochromat objective (1.46 numerical aperture). Images were captured every $1 \mathrm{~s}$ for $2.5 \mathrm{~min}$ at a fixed exposure time across both control and KIFC1-depletion conditions.

Experimental design and statistical analyses. Statistical analyses were conducted and graphs were constructed using Excel (Microsoft) or Prism version 7.0 (GraphPad). Data are shown as mean \pm SEM unless stated otherwise. Box plots always outline distribution of the data. The middle line indicates the median of the data. The box always extends from the 25 th to 75 th percentiles. Upper and lower whiskers of the box plots always represent maximum and minimum of the data, respectively. The whiskers are drawn down to the fifth percentile and up to the $95^{\text {th }}$, but also plot each individual value as a point superimposed on the graph. In each experiment, at least three independent repeats were performed. For statistical analysis, the mean difference was considered to be significant if $p<0.05$. Specific statistical analyses are indicated in the figure legends. All data were checked for normality using the Shapiro-Wilk test. Parametric data were assessed using the $t$ test to compare means and nonparametric data were subjected to the Mann-Whitney $U$ test for comparison of medians. All Western blots were analyzed using Image Lab software. Multiple group comparison was performed by one-way ANOVA followed by Bonferroni post hoc analyses in the case of parametric data and Kruskal-Wallis test in the case of nonparametric data. Microtubule slid-

$\leftarrow$

(Figure legend continued.) Data are represented as mean \pm SEM. Unless otherwise specified, all statistical test comparisons were done against the KIFC1 siRNA group compared with the control siRNA group in the case of depletion or with the KIFC1 siRNA + HSET-GFP and control siRNA + GFP groups in the case of rescue experiments. ANOVA test was used for multiple comparisons and paired comparisons were analyzed using Student's $t$ test. Scale bars: $\boldsymbol{B}$, control siRNA, KIFC1 siRNA, $15 \mu \mathrm{m}$; control siRNA + GFP, KIFC1 siRNA + GFP, $10 \mu \mathrm{m}$. ing data were analyzed using publicly available, open-source plugins in Fiji (National Institutes of Health). Td-Eos-tubulin intensity values were measured for each time point. All morphological analyses were performed using the same software. EB3 comet analyses were performed using Plus Tip Tracker software and MATLAB.

\section{Results}

\section{KIFC1 is expressed in neurons}

In an earlier study on cultured rat hippocampal neurons, the Banker laboratory used quantitative PCR to examine mRNA expression patterns of kinesin superfamily members and found that KIFC1 is the strongest expressed of any of the kinesins that are best known for generating forces on microtubules during mitosis (Silverman et al., 2010). Moreover, unlike in the case of the other force-generating mitotic kinesins, expression of KIFC1 did not taper off during maturation, but remained relatively high in mature cultures. KIFCl's expression in the nervous system has also been documented by in situ hybridization in the Allen Mouse Brain Atlas and by proteomics studies from the Human Protein Atlas project. Studies on KIFC1 protein in rat are hindered by the fact that available antibodies are generated against human protein and thus are not optimized for work on rodents. We found one commercially available KIFC1 antibody that was effective for Western blotting rodent samples, with analyses revealing changes in the levels of the protein during key stages of development of rat hippocampus (Fig. 1A,B). Unlike other mitotic kinesins, KIFCl's expression remained continuously high during the development of the hippocampus and peaked at P10 and P15.

Primary cultures of rat fetal hippocampal neurons or sympathetic neurons from the SCG of newborn rats displayed a prominent KIFC1 band that was reduced by $\sim 72 \%$ in cultures that had been transfected $3 \mathrm{~d}$ prior with a pool of four siRNA sequences specific to rat KIFC1 (Fig. 1C). A different KIFC1 antibody, which proved to be better for immunostaining, stained cultured rat hippocampal neurons in a manner that was diminished by KIFC1 siRNA (Fig. 1D).

\section{KIFC1 depletion or inhibition affects axon formation}

To investigate the functions of KIFC1 in primary neuronal cultures, we studied the effects of siRNA-based depletion of the protein, but we also took another approach, which was to acutely inhibit KIFC1 with two different small-molecule inhibitors of KIFC1's ATPase activity. These two drugs, termed AZ82 and SR31527, are both effective and specific, but (as discussed in more detail in the Discussion section) have somewhat different mechanisms of action (Wu et al., 2013; Yang et al., 2014; Zhang et al., 2016; Park et al., 2017). To avoid nonspecific toxicity of the drugs, neuronal viability was assessed using the MTT assay (3-(4, 5-dimethylthiazolyl-2)-2, 5-diphenyltetrazolium bromide), a colorimetric test for cell viability, for both inhibitors at varying concentrations. These studies as well as preliminary morphological studies were performed to select the lowest (nontoxic) concentration of the inhibitors that produced the maximum morphological deficit: in this case, shortening of axonal length (Fig. $2 A, B$ ). On these bases, we chose 2 and $20 \mu \mathrm{M}$ for AZ82 and SR31527, respectively, for further studies. For siRNA experiments, the pool of siRNA sequences (or control scrambled siRNA sequence) was introduced by nucleofection before plating. Sixty hours after nucleofection, the neurons were replated to allow process outgrowth to occur anew. Control for the drugs, which were added at the time of plating, was the DMSO vehicle into which the drugs were dissolved. Two different kinds of rat neurons were used throughout the studies, fetal hippocampal and 


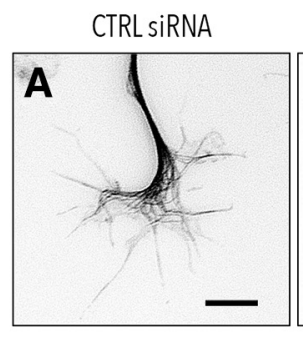

$\mathbf{F}$
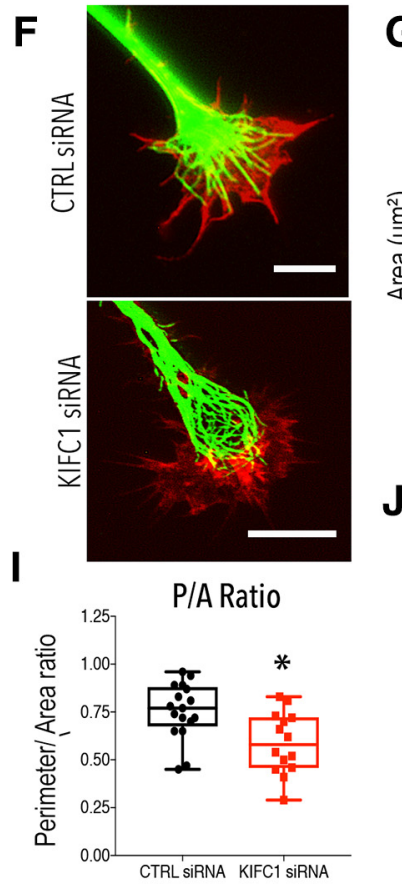

KIFC1 siRNA

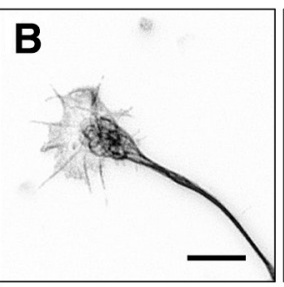

G Area of the growth cone

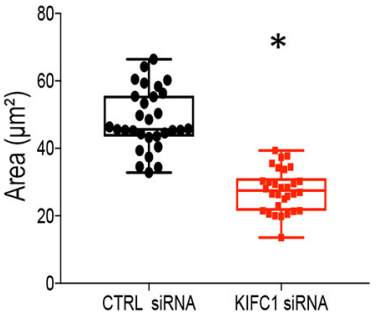

J

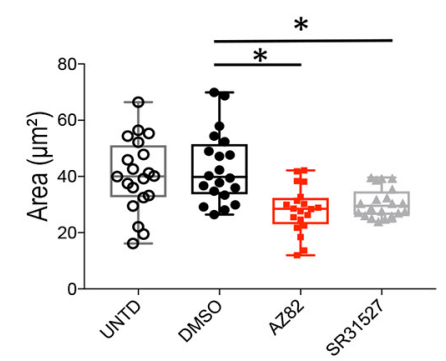

L EB3 track movement
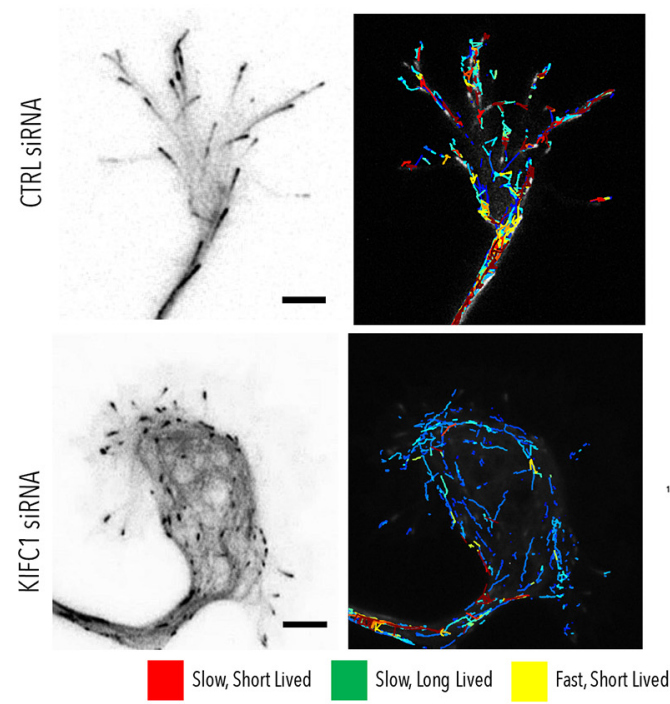

Directionality of EB3 comet

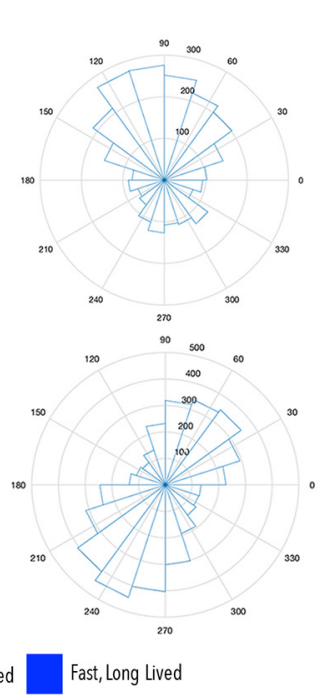

SR31257
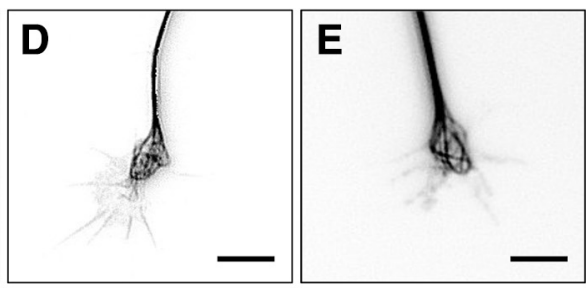

H

Microtubule invasion into filopodia

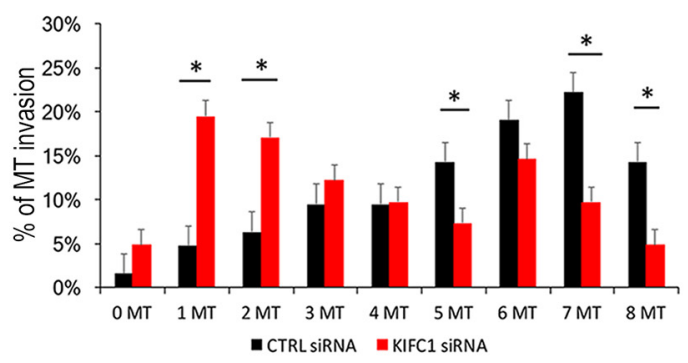

K

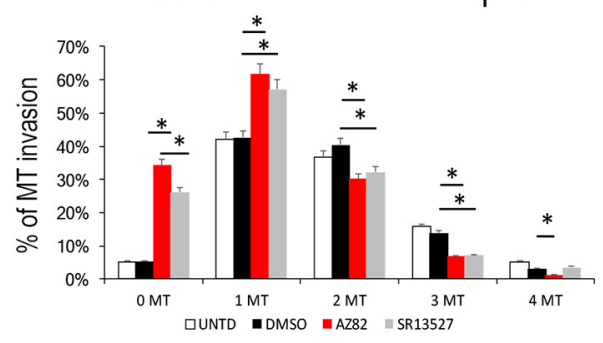

M

EB3 growth lifetime

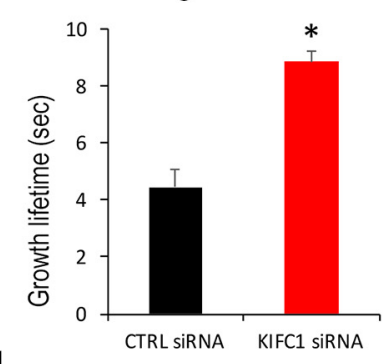

$\mathbf{N}$

EB3 comet speed

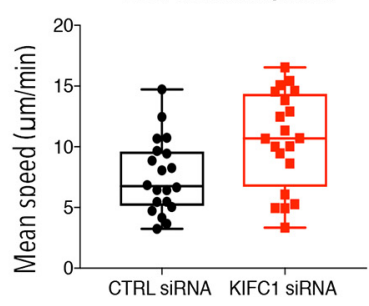

Figure 5. KIFC1 depletion induces changes in growth cone morphology and microtubule organization. $\boldsymbol{A}-\boldsymbol{E}$, Representative fluorescence images (inverted for better contrast) of growth cones of sympathetic (SCG) neurons from control, KIFC1 siRNA, DMSO, SR31527, and AZ82 groups immunostained for $\beta$ III-tubulin. $\boldsymbol{F}$, Representative immunofluorescence images of control siRNA-treated growth cone of sympathetic (SCG) neurons (top) and KIFC1 siRNA treated growth cone of sympathetic (SCG) neurons (bottom). Green, Microtubules; red, actin. $\mathbf{G}$, Box plot representing the quantification of area of growth cone of control siRNA- and KIFC1 siRNA-treated neurons. ${ }^{*} p<0.05 ; n=30$ per group. $\boldsymbol{H}$, Frequency histogram of the number per growth cone of filopodiacontaining microtubules of control and KIFC1-depleted neurons. ${ }^{*} p<0.05 ; n=30$ per group. I, Box plot representing quantification of the ratio of perimeter to area of growth cones of control or KIFC1 siRNA-treated neurons. ${ }^{*} p<0.05$; control siRNA, $n=17$; KIFC1 siRNA, $n=14$. J, Box plot representing quantification of area of growth cone. ${ }^{*} p<0.001 n=20$ per group. $K$, Bar graph average percentage of the number of microtubules invading into filopodia (J) from untreated or DMSO-, SR31527-, or AZ82-treated neurons. ${ }^{*} p<0.05 ; n=20$ per group. $L$, Color-coded microtubule growth track subpopulation overlays from $150 \mathrm{~s}$ time-lapse movies of GFP-EB3. Tracks were overlaid on a radial graph and represented as histogram plots for control growth cone and KIFC1-depleted growth cone. $\boldsymbol{M}, \boldsymbol{N}$, Bar graphs representing quantification of EB3 comet lifetime $(\boldsymbol{M})$ and EB3 comet speed $(\boldsymbol{N})$ of control and KIFC1-depleted (Figure legend continues.) 


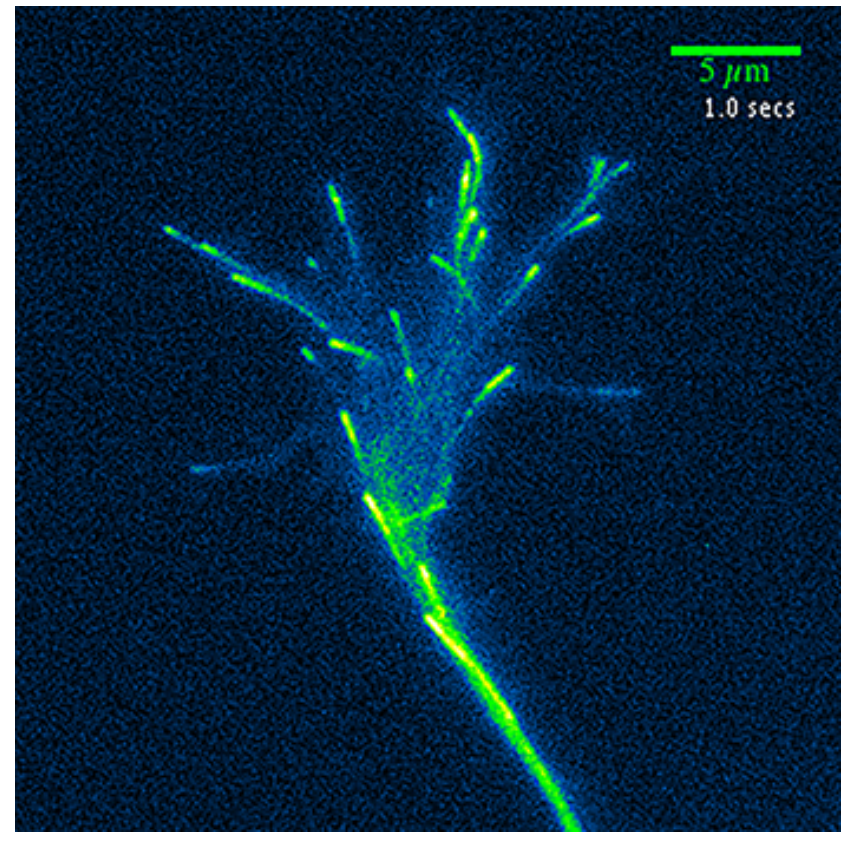

Movie 1. GFP-EB3 comets in control siRNA-treated growth cone. Movie is related to Figure 4 and shows EB3-GFP comet movement in the growth cone of control siRNA-treated neurons pseudocolored with a green-fire blue filter over a period of $150 \mathrm{~s}$. Scale bar, $5 \mu \mathrm{m}$.

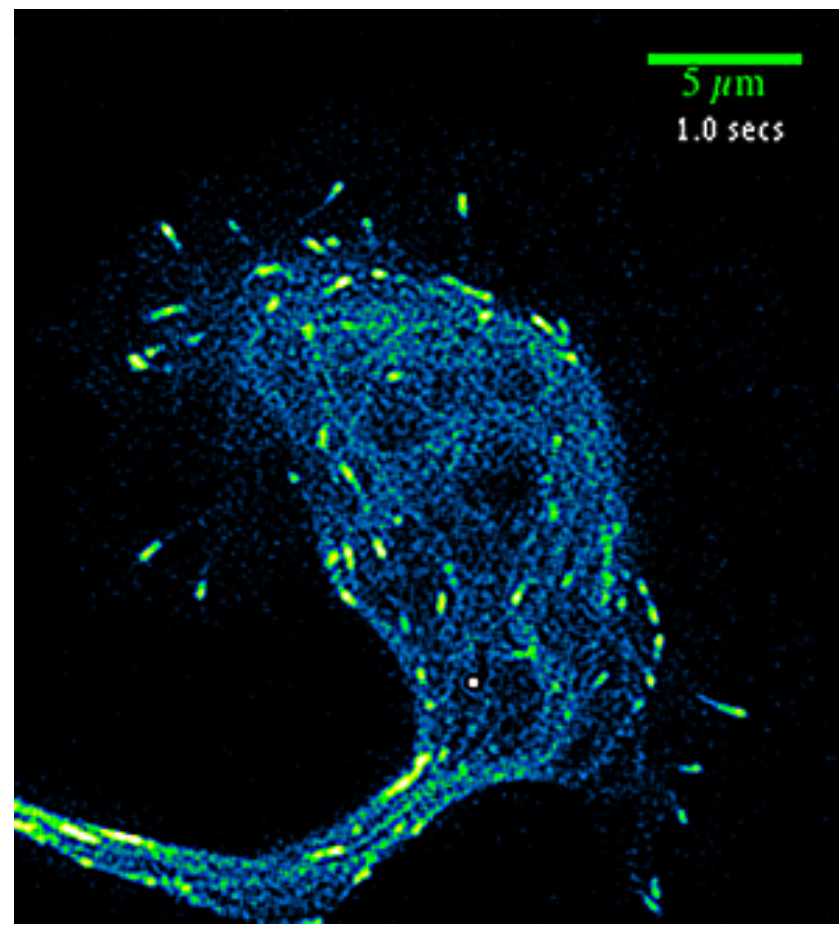

Movie 2. GFP-EB3 comets in KIFC1 siRNA-treated growth cone. Movie is related to Figure 4 and shows EB3-GFP comet movement in the growth cone of KIFC1 siRNA-treated neurons pseudocolored with green-fire blue filter over a period of $150 \mathrm{~s}$. Scale bar, $5 \mu \mathrm{m}$.

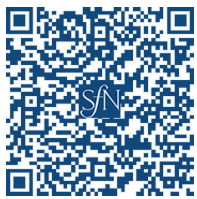

newborn rat (SCG) sympathetic, because each has characteristics appropriate for different kinds of experiments.

When fetal hippocampal neurons are dissociated and then cultured, they reestablish polarity by progressing through well defined stages of development (Banker, 2018). According to the stages defined by Banker's group, stage 1 neurons have lamellae but no processes (Fig. 2C). Stage 2 neurons have several essentially identical minor processes (Fig. 2C). Stage 3 is characterized as axon specification in which one minor process undergoes a period of growth and attains an axonal phenotype (Fig. 2C). Staining for $\beta$ III-tubulin confirmed neuronal identity. At both 24 and $48 \mathrm{~h}$, KIFC1-depleted neurons continued to stain for $\beta I I I-$ tubulin and thus did not switch to a different cellular fate. Rather, KIFC1-depleted neurons and KIFC1-inhibited neurons were found, on average, to lag behind their control counterparts in their progression through these stages (Fig. $2 C-E^{\prime}$ ). In addition, KIFC1-depleted neurons as well as KIFC1-inhibited neurons had relatively shorter mean axonal length compared with stage 3 neurons (Fig. $3 A-C$ ). This was shown by quantification of the length of the primary axon relative to controls (Fig. $3 D, E$ ) in KIFC1depleted neurons and KIFC-inhibited neurons, as well as of secondary and tertiary axons (i.e., axonal branches; data not shown). Because KIFC1 protein remaining after siRNA treatment was detected only in the cell body, we were able to perform an additional experiment to ascertain the specificity of the drugs. Neurons de-

$\leftarrow$

(Figure legend continued.) hippocampal growth cones. ${ }^{*} p<0.05, n=25$ per group. Error bars indicate SEM. Unless otherwise specified, all statistical test comparisons were done against control siRNA group with KIFC1 siRNA group in case of depletion using Student's $t$ test. In the case of drug treatments, the DMSO group was compared with the untreated, AZ82, or SR31527 groups using ANOVA test. Further multiple comparisons were made using Bonferroni test. Scale bars: $\boldsymbol{A}-\boldsymbol{E}, 10 \mu \mathrm{m} ; \boldsymbol{F}, 15 \mu \mathrm{m} ; \boldsymbol{L}, 5 \mu \mathrm{m}$. no additional changes in their axons, confirming the specificity of the drugs (Fig. $3 F$ ). We also quantified the frequency of branching (i.e., axonal branch number) of both control and KIFC1depleted neurons and observed a decrease in branching frequency in the axons of KIFC1-depleted neurons (Fig. 3G) and this same result was obtained with the drugs (Fig. $3 H$ ). For these and all morphological studies, cultures were immunostained for $\beta$ III-tubulin and the images were displayed as "inverted images" for better contrast.

Finally, we turned to cultures of rat sympathetic (SCG) neurons for live-cell imaging of axonal growth because the axons of these neurons grow extremely rapidly in response to laminin, whereas the axons of cultured hippocampal neurons are relatively slow growing, even when exposed to laminin. Unlike hippocampal neurons, these neurons do not undergo stereotyped stages of development, but rather extend over the first few days of culture multiple processes, all of which are bona fide axons. We generated movies of axons growing over a $3 \mathrm{~h}$ period of time under phase-contrast microscopy and observed that the KIFC1depleted axons grew in spurts with intermittent periods of slow growth, whereas control axons grew more steadily with only minimal stalling during the same period of time. Because of some variability in this, we quantified traces of individual axons during their growth phase (Fig. 3I-K).

\section{KIFC1 depletion induces microtubule polarity flaws in the axon}

We next investigated the relevance of KIFC1 to microtubule organization in the axon of cultured rat hippocampal neurons by observing end-binding protein 3 tagged with GFP (EB3-GFP), pleted of KIFC1 and then exposed to the KIFC1 drugs displayed 
A

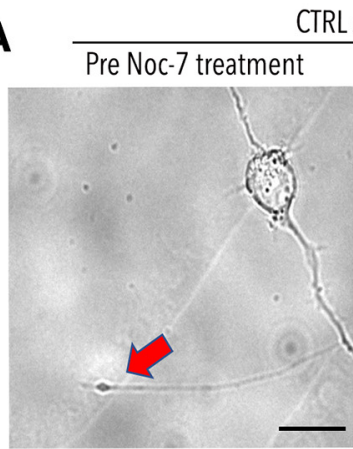

B

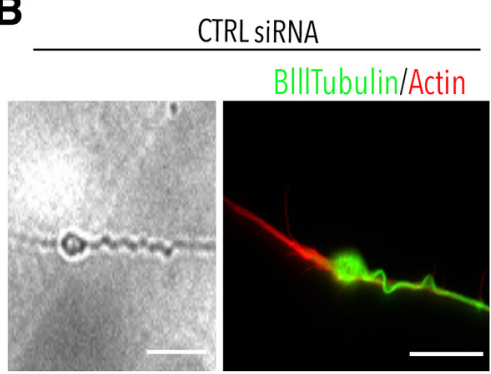

Post Noc-7 treatment
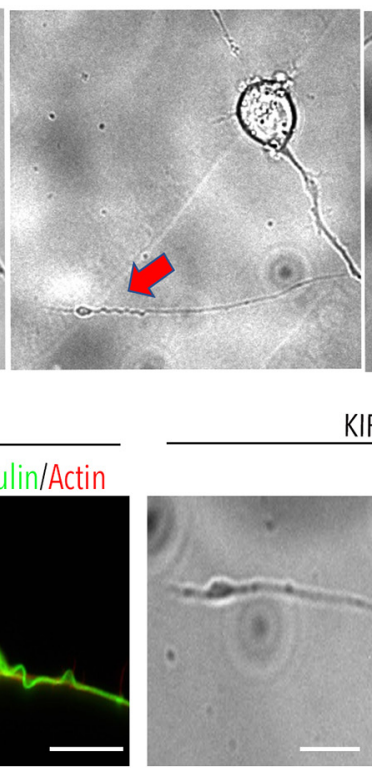

KIFC1 siRNA

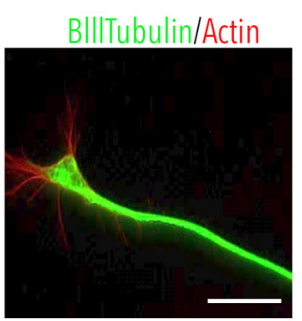

KIFC1 SiRNA

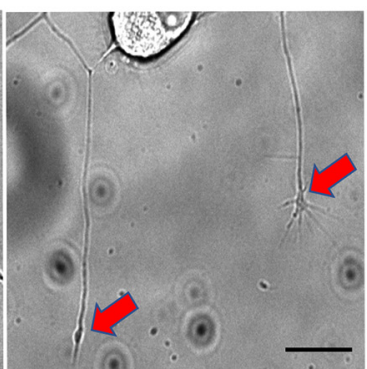

C Axonal retraction length

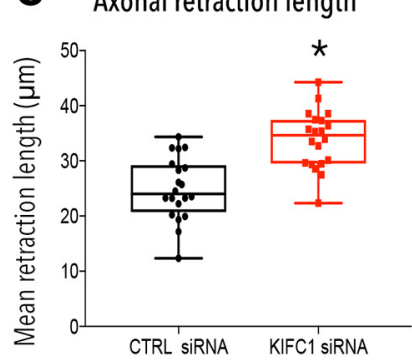

Figure 6. KIFC1 prevents axonal retraction by opposing sliding of long microtubules in the axon. $A$, Representative DIC images of control or KIFC1-depleted sympathetic (SCG) neurons before and after 30 min of NOC-7 treatment. B, DIC and immunofluorescence images of the distal axon of control and KIFC1-depleted sympathetic (SCG) neurons after NOC-7 treatment. Green, Microtubules; red, actin. C, Quantification of mean retraction length of control and KIFC1-depleted neurons after NOC-7 treatment are represented as boxplot. ${ }^{*} p<0.001 ; n=20$ per group. Data are represented as mean \pm SEM. Unless otherwise specified, all statistical test comparisons were done against the control siRNA group/DMSO with KIFC1 siRNA/AZ82 group using Student's $t$ test. Scale bars: $A$, 50 $\mu \mathrm{m}, \boldsymbol{B}, 20 \mu \mathrm{m}$.

which manifests as comet-shaped excursions at the plus end of the microtubule, representing bouts of assembly. The directionality of the excursion reveals the polarity orientation of the microtubule. A "polarity flaw" is defined as a minus-end-out microtubule in the axon. The entire length of the axon was traced to analyze microtubule behaviors. All EB3-GFP trajectories for $150 \mathrm{~s}$ bouts of imaging were mapped and overlaid as histograms over the radial plots (Fig. 4A). We also traced the axons and generated kymographs to assist in identifying microtubule polarity flaws along the length of the axon. Traced kymographs (see next paragraph for details on experiments) indicate that polarity flaws exist all along the length of the KIFC1-depleted axon but are especially high in its distal third, and that these effects were rescued when HSET+GFP was ectopically expressed (Fig. 4B).

Quantification showed that $\sim 22 \%$ of the observed trajectories in the entire KIFC1-depleted axon were directed toward the cell body (indicating minus-end-out microtubules) compared with $\sim 4 \%$ in control axons (Fig. 4 C). Mapping the EB3-GFP trajectories in the distal third of the axon's length (Fig. $4 D$ ), the polarity flaws were more severe, with $\sim 38 \%$ of the observed trajectories in KIFC1-depleted axons directed toward the cell body, compared with $\sim 8 \%$ in control axons. This means that the rest of the axon (not including the distal third) consisted of $\sim 12 \%$ of the observed trajectories in KIFC1-depleted axons directed toward the cell body compared with $\sim 4 \%$ in control axons (Fig. $4 E$ ). These results indicate significant flaws in microtubule polarity orientation in the axon (in which microtubules are normally almost entirely plus-end-out) that arise when KIFC1 is depleted, with the flaws especially abundant in the distal third of the axon. We also observed differences in mean speed of the EB3-GFP comets, with faster-moving comets that had a longer lifetime in KIFC1depleted axons compared with control axons (Fig. 4F, G). Rescue experiments with ectopically-expressed HSET (human wild-type
KIFC1, which is not affected by the siRNA for rat KIFC1) corrected the microtubule polarity flaws (Fig. $4 C$ ) and restored the comet speed and comet lifetime to normal, demonstrating the specificity of the effects to the KIFC1 depletion (Fig. $4 F, G$ ). Entirely similar results were obtained in a smaller number of studies on rat sympathetic (SCG) neurons (data not shown).

\section{KIFC1 depletion induces growth cone collapse}

The tips of KIFC1-depleted axons (of both types of neurons) were blunter than their control counterparts and more club shaped (Fig. 5A,B). In fixed neurons fluorescently stained for microtubules and actin filaments, the microtubules were notably more curled in KIFC1-depleted growth cones than control growth cones. This phenomenon was also observed in neurons treated with either of the two KIFC1 drugs (Fig. 5C-E). The curled microtubules in KIFC1-depleted growth cones were akin to those in stalled growth cones or growth cones of retracting axons (Szebenyi et al., 1998; Dent et al., 1999), whereas the microtubules in control growth cones were spread in a fan-shaped manner, with some entering filopodia. Quantification of the area of the growth cone revealed that KIFC1-depleted neurons had smaller growth cones (Fig. $5 F, G$ ) and a smaller percentage of filopodia occupied by microtubules compared with controls (Fig. $5 \mathrm{~F}$ and $5 \mathrm{H}$ ). The ratio of the perimeter to area of the growth cone was significantly smaller in KIFC1-depleted neurons compared with control growth cones (Fig. 5I). Entirely similar effects on the area of the growth cone and microtubule invasion into filopodia were observed in neurons treated with AZ82 or SR31527 (Fig. 5J,K).

To further examine microtubule behaviors in the growth cone, we transfected hippocampal neurons of control and KIFC1-depleted groups to express EB3-GFP. We chose to do these studies on hippocampal neurons because they have larger growth cones. In the growth cones of control neurons, EB3 comets predominantly moved in relatively straight trajectories to- 


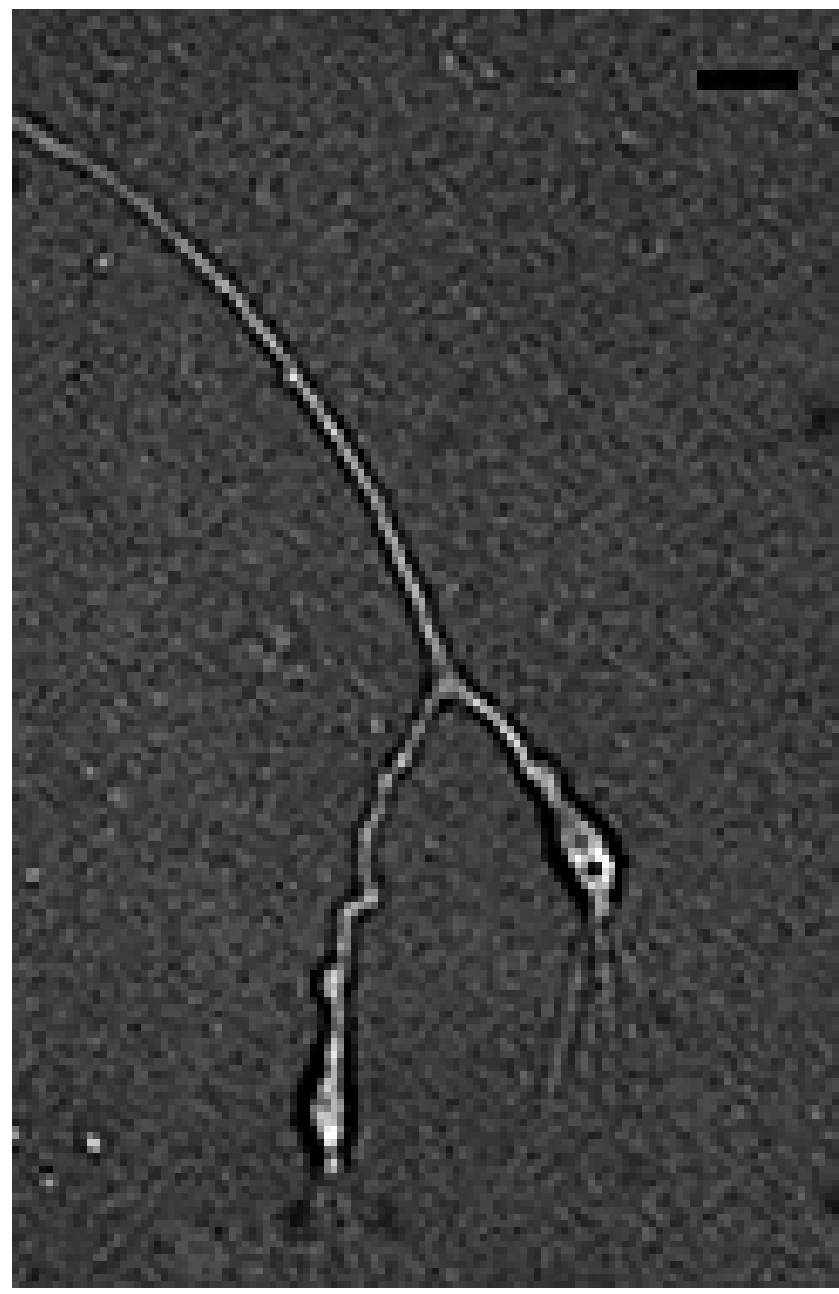

Movie 3. Axonal retraction induced by NOC-7 (control siRNA. Movie is related to Figure 6 and is a phase-contrast movie of the growth cone of control siRNA-treated neurons subjected to NOC-7. Frames are shown every $5 \mathrm{~min}$ for a period of $30 \mathrm{~min}$. Scale bar, $10 \mu \mathrm{m}$.

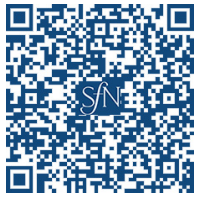

ward the leading edge into the filopodia (Fig. 5 L, Movie 1). In contrast, after KIFC1 depletion, EB3 comet tracks did not follow one another and their paths failed to orient toward the leading edge (Fig. 5L, Movie 2). Rather, the comets moved in curled trajectories that often became directed back into the axonal shaft, probably accounting for at least some of the observed microtubule polarity flaws in that region of the axon. At the neck of the growth cone, comet trajectories were not aligned with one another as well as in controls (Fig. 5L). EB3 comet lifetime and speed increased in KIFC1-depleted neurons compared with control neurons (Fig. 4M,N), indicating that the microtubules had become more dynamic as a result of KIFC1 depletion. Figure $4 L$ is displayed using plusTipTracker software (Applegate et al., 2011; Stout et al., 2014), in which the magnitudes of comet speed and lifetime are color coded to allow qualitative visualization of regional differences in these values.

\section{KIFC1 prevents axonal retraction}

We hypothesized that KIFC1 might exist in the axon, especially the distal third of the axon, to fortify it against retraction, thereby enabling the axon to grow steadily. If this is correct, then we predicted that factors that induce retraction of the axon would do

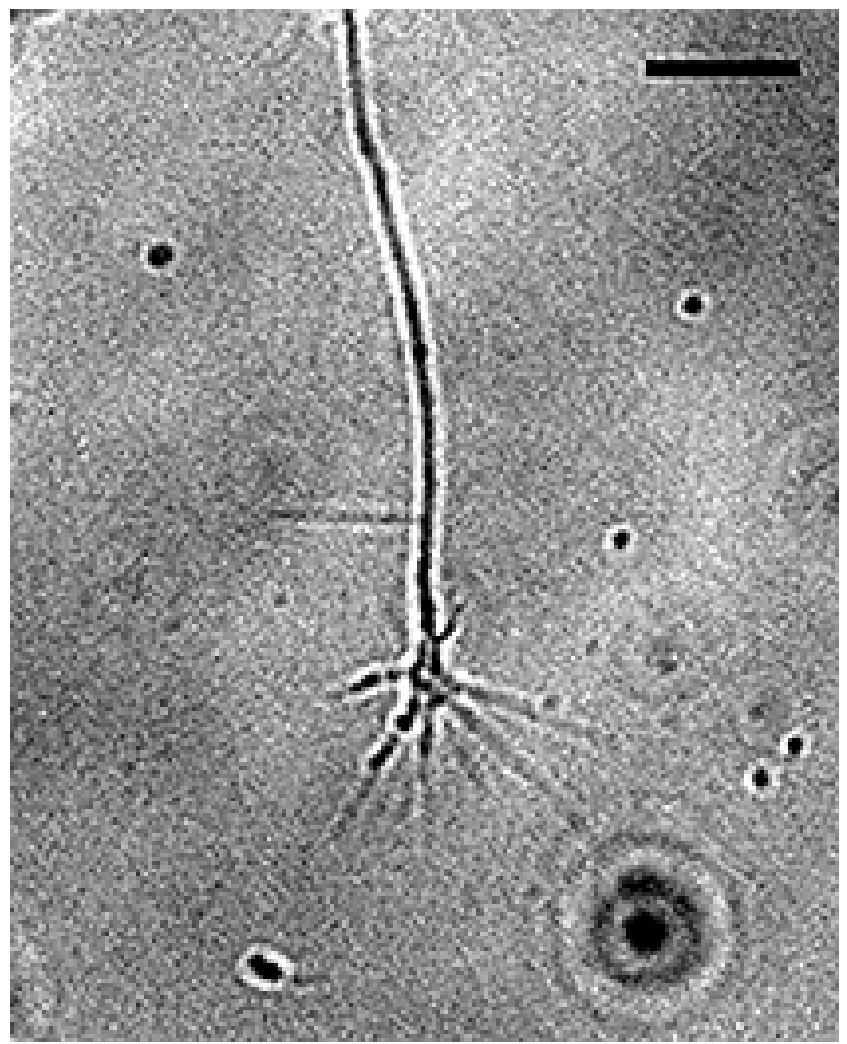

Movie 4. Axonal retraction induced by NOC-7 (KIFC1 siRNA. Movie is related to Figure 6 and is a phase-contrast movie of the growth cone of control siRNA-treated neurons subjected to NOC-7. Frames are shown every $5 \mathrm{~min}$ for a period of $30 \mathrm{~min}$. Scale bar, $10 \mu \mathrm{M}$.

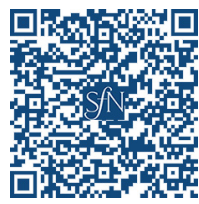

so in a more robust fashion in the absence of KIFC1. To test this, sympathetic (SCG) neurons depleted of KIFC1 were exposed to NOC-7 (a nitric oxide donor that induces retraction of axons of SCG neurons; see He et al., 2002). Studies were conducted on neurons fixed after $30 \mathrm{~min}$ of exposure to NOC-7 and then prepared for fluorescence visualization of microtubules and actin filaments and on living neurons during the NOC-7-induced retraction of the axon observed with DIC microscopy. Consistent with our prediction, DIC images of the distal third of the axon indicated that KIFC1-depleted axons retracted more extensively in response to NOC-7 than control axons and also with a very different morphology of retraction (Fig. 6A-C). Control axons displayed sinusoidal bends (Movie 3), as has been reported repeatedly in the past of axons induced to retract by various stimuli (He et al., 2002; Myers and Baas, 2007), but no such bends were observed in the case of KIFC1-depleted axons (Fig. 6B, Movie 4). The sinusoidal bends are caused by long microtubules that do not slide back paraxially as the axon retracts, so the lack of such bends in KIFC1-depleted axons is consistent with the microtubules in the axon sliding back more readily as the axon retracts.

\section{KIFC1 affects the ratio of anterograde to retrograde transport} of short microtubules in the axon

Microtubules shorter than $10 \mu \mathrm{m}$ are known to be transported rapidly in both directions in the axon, with $\sim 70 \%$ moving anterogradely and the rest moving retrogradely. Cytoplasmic dynein is the chief motor responsible for the bidirectional transport of these microtubules (He et al., 2005; Rao et al., 2017), although 
A

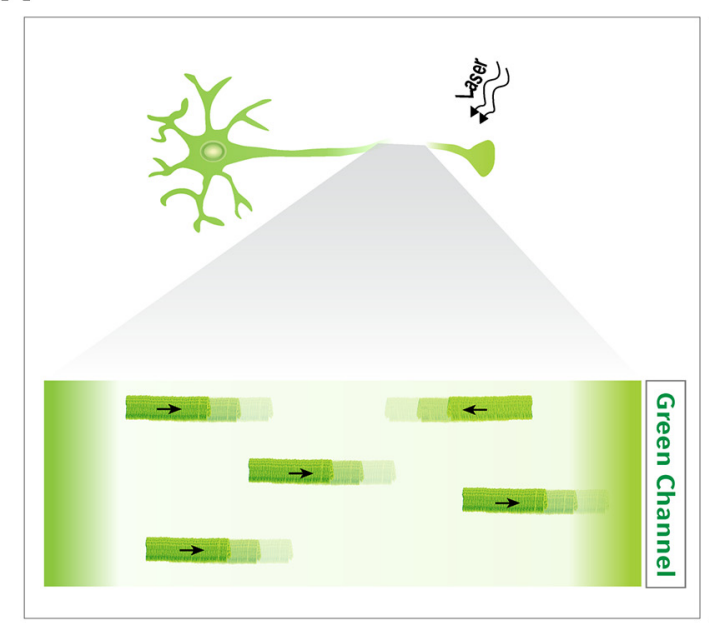

B

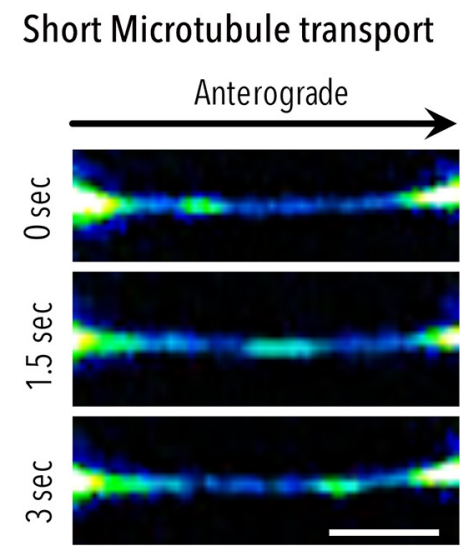

D

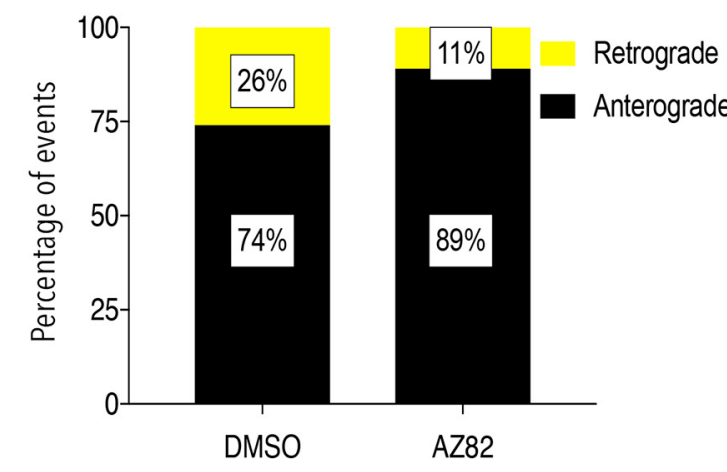

C Direction of short microtubule transport

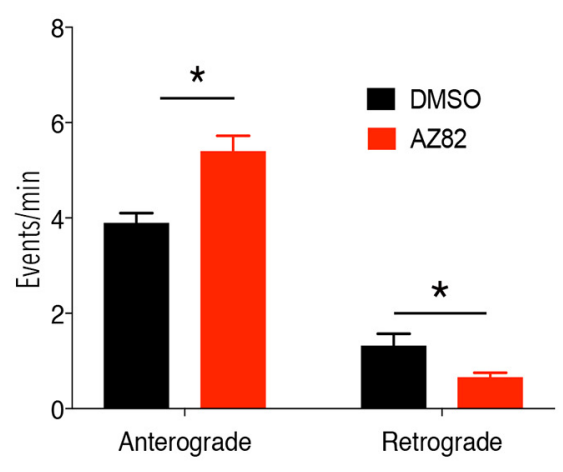

E
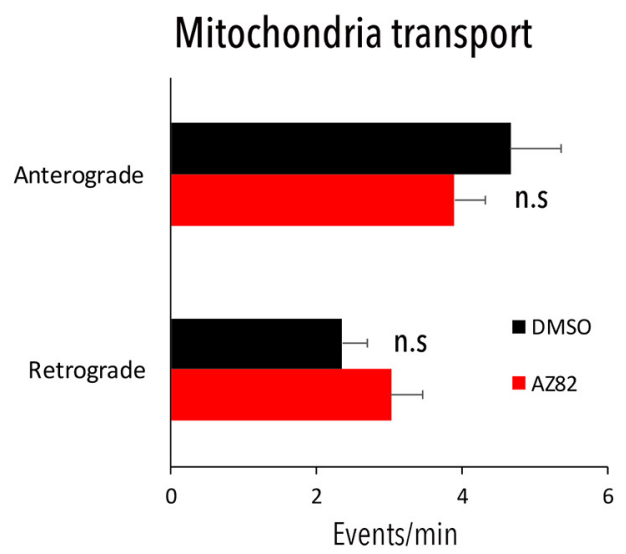

Figure 7. KIFC1 affects the ratio of anterograde to retrograde transport of short microtubules in the axon. $\boldsymbol{A}$, Schematic illustration of the short microtubule transport assay (left) and the microtubule sliding assay (right). Both assays use essentially the same procedure except, to visualize short microtubules moving rapidly, we use the green channel in which short microtubules can be observed to move rapidly from the regions flanking the photoconverted zone through the photoconverted zone. To visualize evidence of long microtubules sliding, we use the red channel and look for the red fluorescence of the photoconverted zone to gradually spread into the flanking regions. The data from the latter are shown later in Figure $9 . \mathbf{B}$, Representative fluorescence image of short microtubule transport in the distal third of the axon. $C$, Bar graph representing quantification of average short microtubule transport events per minute in the distal third of the axon from DMSO- and AZ82-treated sympathetic (SCG) neurons. ${ }^{*} p<0.05$. DMSO, $n=15 ;$ AZ82, $n=12$. D, Stacked graph representing quantification of the frequencies of anterograde and retrograde microtubule transport events in DMSO- and AZ82-treated neurons. DMSO, $n=15 ; \mathrm{AZZ2}, n=12$. E, Bar graph representing quantification of the number of axonal mitochondria moving in control and KIFC1 siRNA groups. $n=12$ per group. Data are represented as mean \pm SEM. Unless otherwise specified, all statistical test comparisons were done against the control siRNA group/DMSO with the KIFC1 siRNA/AZ82 group using Student's $t$ test. Scale bar in $B, 10 \mu \mathrm{m}$. 
A

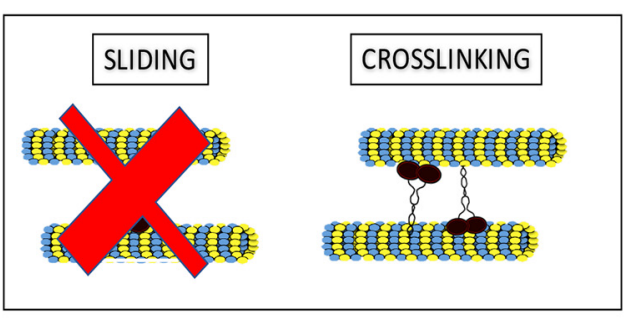

D
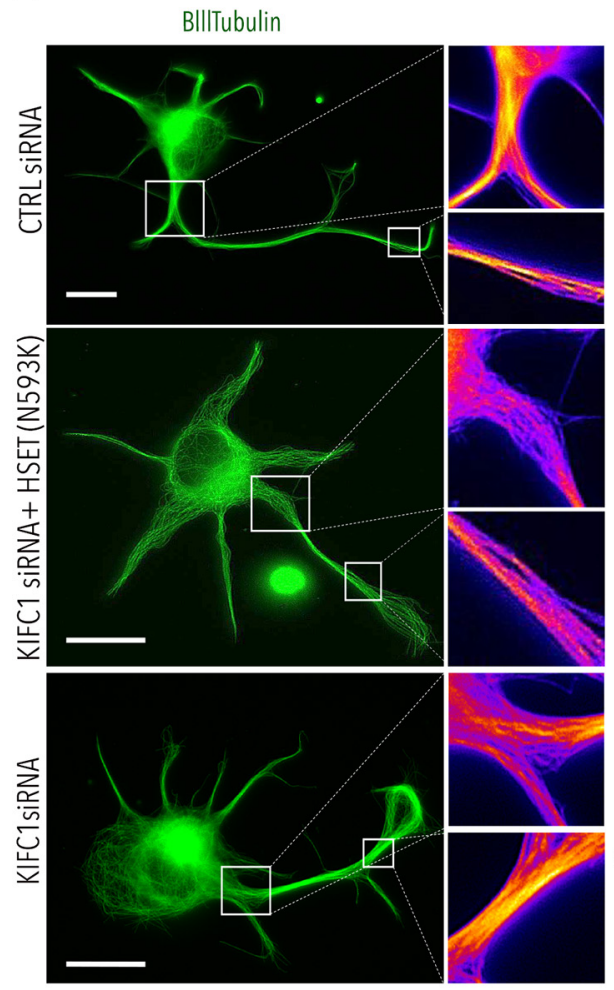

J

E
B

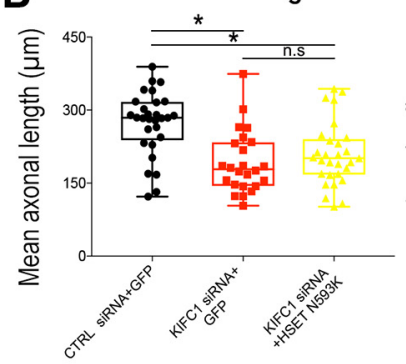

C Growth cone

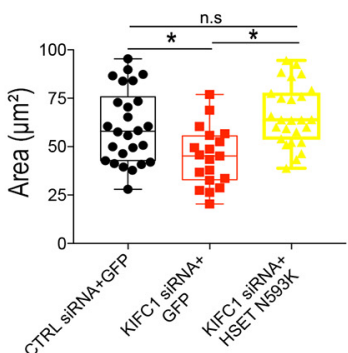

KIFC1 siRNA
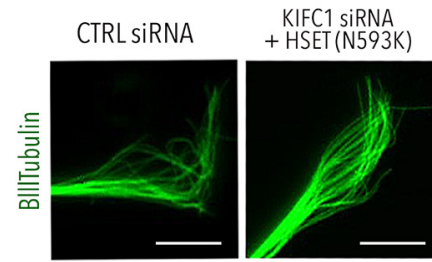

F

Proximal axon

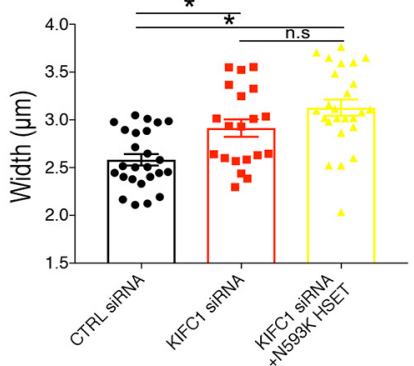

G

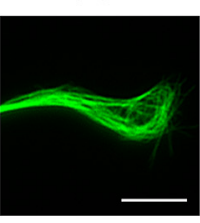

Distal Axon

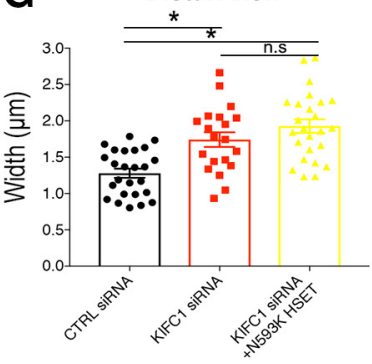

H
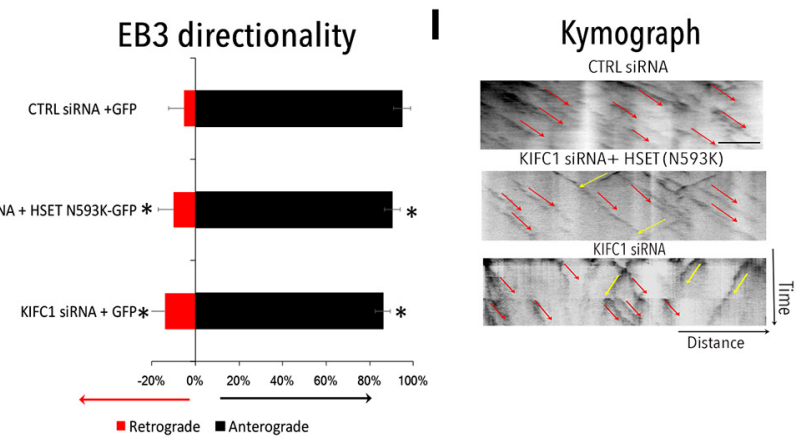

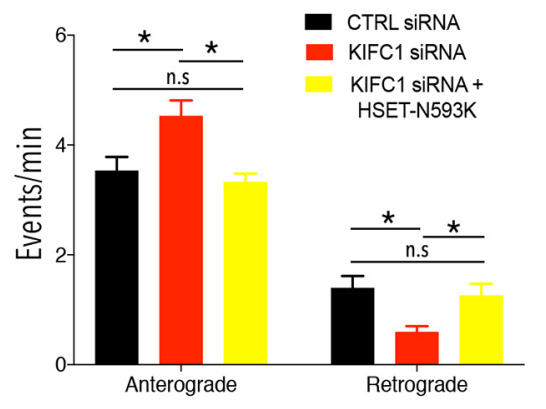

K

Total number of events

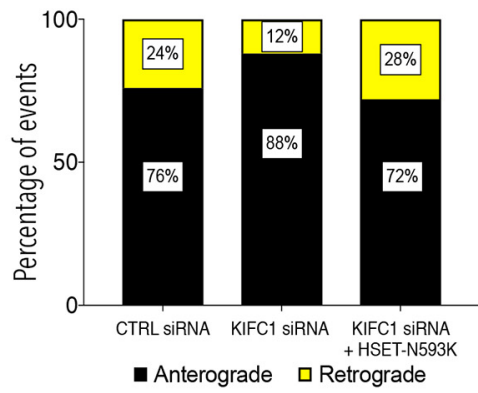

Figure 8. Studies with KIFC1 mutant that cross-links but does not slide microtubules. $A$, Schematic illustration of the KIFC1 cross-linking-only mutant's inability to slide microtubules while remaining capable of cross-linking microtubules in a relatively normal manner. $\boldsymbol{B}, \boldsymbol{C}$, Box plots represent the mean axonal length $\left({ }^{*} p<0.05 ; \boldsymbol{B}\right)$ and area of the growth cone $(\boldsymbol{C})$ from control neurons and neurons ectopically expressing the cross-linking-only mutant along with KIFC1-depleted neurons. ${ }^{*} p<0.05, n=29$ per group except KIFC1 siRNA group, where $n=24$. D, Representative immunofluorescence images staining for microtubules of control and cross-linking-only mutant expressing neurons and KIFC1-depleted neuron; insets of pseudocolored immunofluorescence images showing the wider-spaced microtubule effect seen in proximal and distal axons of mutant-expressing hippocampal neurons compared with controls. The pseudocolored fire scale filter was used to enhance the qualitative visualization of the spacing of microtubules in the proximal and distal regions of the axon. The bright orange-red color indicates bundled microtubules and the magenta color indicates microtubules being splayed. $\boldsymbol{E}$, Representative immun ofluorescence images of the growth cone of control neurons and mutant-expressing neurons stained for microtubules. $\boldsymbol{F}, \boldsymbol{G}$, Bar graph representing the quantification of the average width of the proximal axon $(\boldsymbol{F})$ and distal axon $(\boldsymbol{G})$ of control and mutant-expressing neurons. ${ }^{*} p<0.05, n=25$ except KIFC1 siRNA, where $n=20 . \boldsymbol{H}$, Bar graph of quantification of percentage of EB3 comet movement in the entire length of the axon of control and mutant-KIFC1-expressing hippocampal neurons. ${ }^{*} p<0.05, n=$ 25 per group except KIFC1 siRNA, where $n=20$. I, Representative kymographs of control siRNA-treated and KIFC1 siRNA+cross-linking-only mutant treated (Figure legend continues.) 
a number of other motors also influences their transport, for example, by acting as brakes (Myers and Baas, 2007; Liu et al., 2010; Lin et al., 2012; Del Castillo et al., 2015). Acute inhibition of a molecular motor is the best way to reveal its role in microtubule transport given that prolonged depletion of a motor by RNAi can result in changes in the polarity orientation of both short mobile microtubules and the longer microtubules against which the short ones are transported and can also allow motors that normally do not transport short microtubules to do so (Rao and Baas, 2018). Our various studies have validated the smallmolecule inhibitors of KIFC1 as effective, specific, and nontoxic and thus are suitable to analyze the role of KIFC1 in the transport of short microtubules in the axon. We originally posited that KIFC1 may serve as a backup to cytoplasmic dynein (Baas et al., 2006), in which case a diminution in the numbers of short microtubules moving in both directions in the axon would be predicted of KIFC1 inhibition. To explore this, we transfected sympathetic (SCG) neurons (Myers and Baas, 2007; Rao et al., 2017; the type of neuron we have mainly used in past studies on microtubule transport/sliding in the axon) to express Td-Eostubulin and allowed the cells to grow axons for $14 \mathrm{~h}$. In the case of drug treatment, we added AZ82 or DMSO vehicle/control just before imaging. Td-Eos is a protein that fluoresces in the green channel, but when bleached in the green channel, it fluoresces red in the red channel. For the imaging, an $\sim 30 \mu \mathrm{m}$ region in the middle third of the axon's length was bleached/photoconverted and then short fluorescent microtubules were visualized (in the green channel) moving from the flanking regions through the bleached/photoconverted zone (Fig. $7 A, B$ ). Anterograde transport (Fig. $7 C$ ) events were higher in the presence of AZ82 (5.42 \pm 0.334 events per minute) compared with DMSO-treated controls (3.91 \pm 0.20 events per minute), whereas retrograde transport events were lower in the presence of AZ82 (0.66 \pm 0.09 events per minute) compared with DMSO-treated control (1.32 \pm 0.25 events per minute) neurons. Therefore, there was no change in the total number of short microtubules moving through the photoconverted zone, but there was a marked increase in the proportion of the total moving anterogradely (Fig. 7D). No effects on mitochondrial transport were observed with AZ82, consistent with these results being specific to microtubule transport (as opposed to affecting transport of vesicular cargoes; Fig. $7 E$ ). Interestingly, the bleached/photoconverted zone itself, which shows slight anterograde movement in control axons, displayed somewhat greater anterograde movement in axons in which KIFC1 was either inhibited or depleted (see below).

\section{Studies with the KFC1 cross-linking-only mutant suggest a unified explanation for KIFC1's mechanism in the axon}

Because KIFC1 has the duo abilities to cross-link and slide microtubules, we wanted to better understand how each of these properties contributes to the inhibition/depletion phenotype of

\footnotetext{
$\leftarrow$

(Figure legend continued.) neurons.J, Bar graph representing quantification of average short microtubule transport events per minute in the distal axon from control siRNA- and KIFC1 siRNA-treated neurons and the cross-linking-only mutant expressing neurons. ${ }^{*} p<0.05 . n=$ 15 per group except KIFC1 siRNA, where $n=10 . \boldsymbol{K}$, Stacked graph representing quantification of the frequencies of anterograde and retrograde event in control siRNA-treated, KIFC1 siRNAtreated, and HSET N593K-expressing neurons. $n=15$ per group except KIFC1 siRNA, where $n=20$. Data are represented as means \pm SEM. Unless otherwise specified, all statistical test comparisons were done against the control siRNA group with KIFC1 siRNA and/or KIFC1 siRNA + cross-linking-only mutant group using Student's $t$ test for paired groups and ANOVA test for multiple comparisons. Scale bars: $\boldsymbol{D}, 25 \mu \mathrm{m} ; \boldsymbol{E}, 15 \mu \mathrm{m} ; \boldsymbol{I}, 15 \mu \mathrm{m}$.
}

KIFC1 and thereby gain insight into its mechanism. To investigate this, we conducted additional studies using a mutant form of KIFC1 (termed cross-linking-only mutant) that does not hydrolyze ATP, but preserves its wild-type cross-linking properties (Cai et al., 2009) (Fig. 8A). For these studies and all of our studies with KIFC1 mutants, neurons were depleted of endogenous KIFC1 for $36 \mathrm{~h}$ rather than $60 \mathrm{~h}$ so that a greater portion of the wild-type KIFC1 was present. This is because some mutant forms may need to dimerize with wild-type KIFC1 to interact with the microtubule, thus eliciting a meaningful result. Neurons depleted of KIFC1 but expressing the mutant had shorter axonal length than controls but showed similar size of growth cones to controls (Fig. $8 B-E)$. Unlike axons of control neurons, in which the microtubules in these regions of the axon were spaced more closely (Fig. $8 D$ ), axons depleted of endogenous KIFC1 but expressing the mutant displayed microtubules in their distal and proximal thirds that were more widely spaced (Fig. $8 F, G$ ). The percentage of EB3-GFP comets in the experimental axons was $\sim 12 \%$ moving toward the cell body compared with $\sim 3 \%$ in controls, which is a partial correction relative to the depletion alone without the mutant (Fig. 8H). Kymographs of the entire axonal length reveal that polarity flaws were concentrated in the middle third of the axon (Fig. 8I), indicating that a significant proportion of the polarity flaws enriched in the distal third of KIFC1-depleted axons was corrected by the mutant.

Observations of shorter axonal length and microtubule polarity flaws in neurons depleted of wild-type KIFC1, but expressing the mutant led us to investigate short microtubule transport in the presence of the cross-linking-only mutant. Consistent with the results of the drug studies, quantification revealed that there was an increase of short microtubule transport in the anterograde direction ( $4.53 \pm 0.28$ events per minute) in KIFC1-depleted neurons compared with control siRNA-treated neurons (3.53 \pm 0.25 events per minute) and a decrease in short microtubule transport in KIFC1-depleted ( $0.6 \pm 0.101$ events per minute) neurons compared with controls ( $1.14 \pm 0.21$ events per minute) (Fig. $8 J$ ). These effects were rescued when neurons depleted of wild-type KIFC1 expressed the cross-linking-only mutant (Fig. $8 J)$. Similar to the drug studies, there was no change in the total frequencies of short microtubule transport with any of these manipulations (Fig. $8 \mathrm{~K}$ ).

Therefore, the cross-linking properties of KIFC1, on their own without the ATPase properties, are able to account for a great deal of what KIFC1 does in the axon, but not everything, with the ATPase properties crucial for normal axonal growth, preservation of microtubule polarity orientation in the middle third of the axon, and normal spacing between neighboring microtubules.

\section{KIFC1 opposes sliding of long microtubules in the axon}

Because KIFC1 has been shown to cross-link microtubules and prevent sliding in other experimental scenarios, we hypothesized that an increase in the capacity of the long microtubules in the axon to slide might explain the observed effects of KIFC1 depletion on axonal retraction and growth cones. To explore this, we transfected sympathetic (SCG) neurons (Myers and Baas, 2007; Rao et al., 2017; the type of neuron we have mainly used in past studies on microtubule transport/sliding in the axon) with TdEos-tubulin and then photoconverted the Td-Eos in the distal third of the axon such that the green fluorescence of the probe, when bleached (in the green channel), turns red (in the red channel) in the photoconverted zone (Fig. 7A). The photoconverted fluorescence was quantitatively measured in the red channel 
A
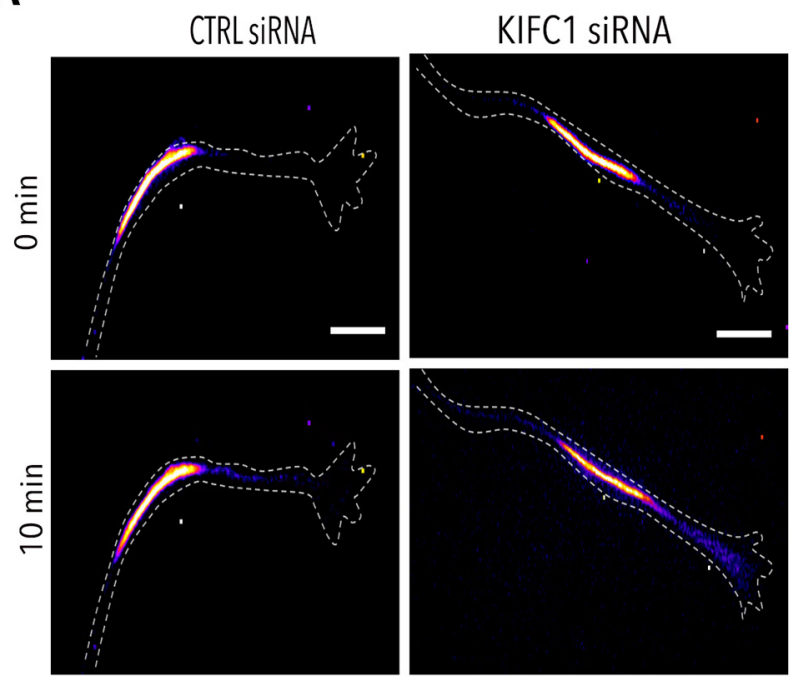

C

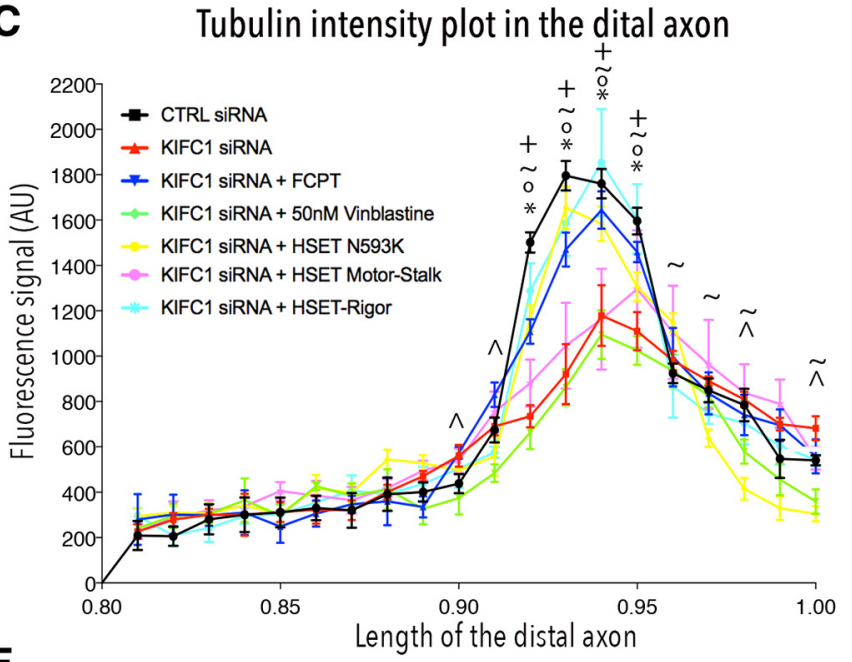

$\mathbf{F}$

CTRL SIRNA

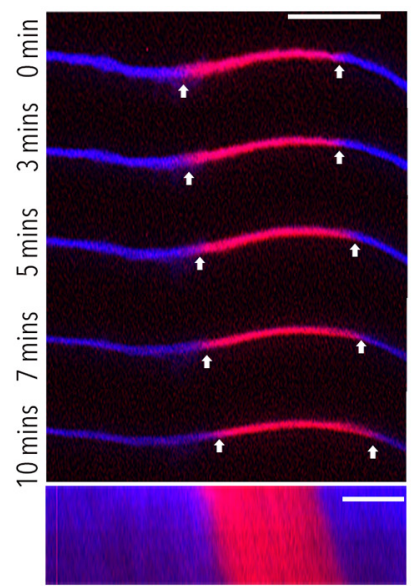

KIFC1 SiRNA

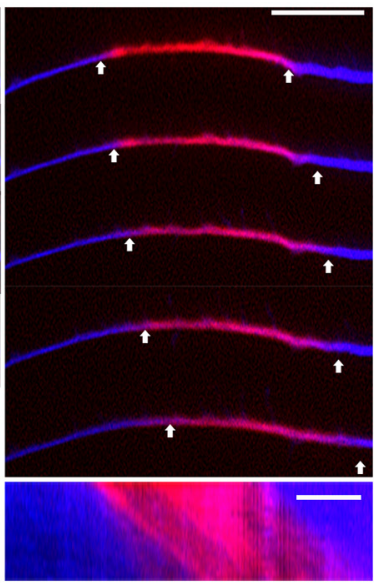

B

D

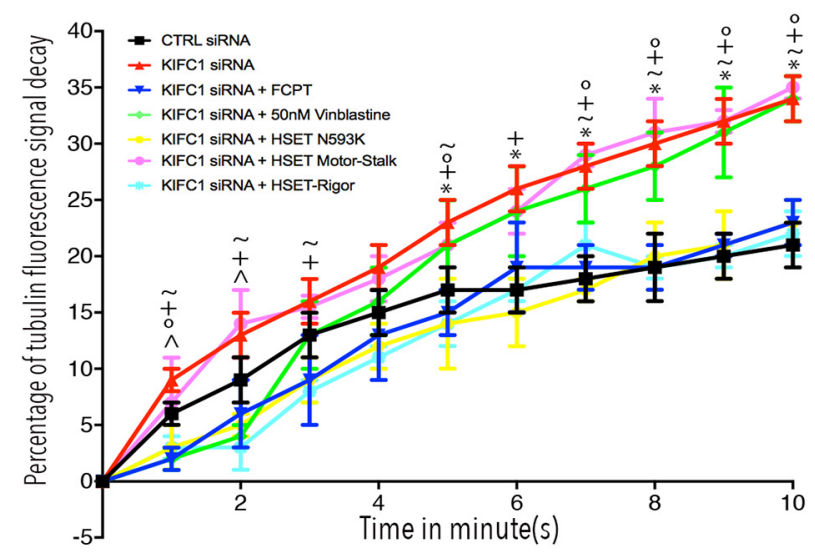

Decay of fluorescence signal

E Width of the peak
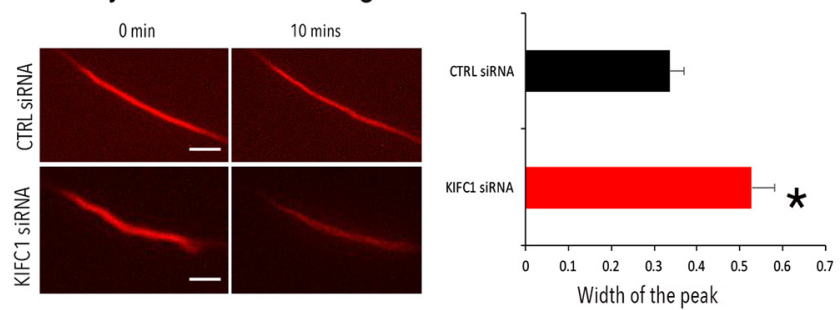

G WILD TYPE

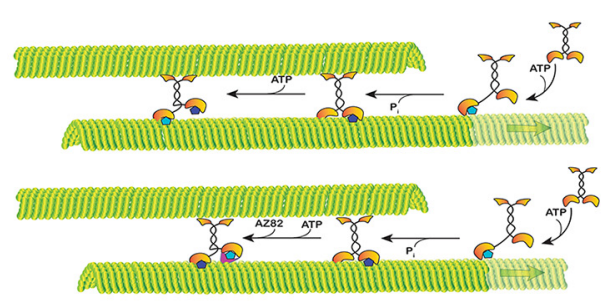

SR31257

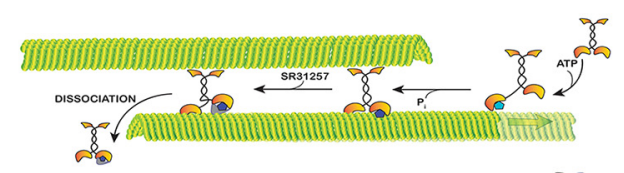

HSET N593K
(Crosslinking only
mutant)

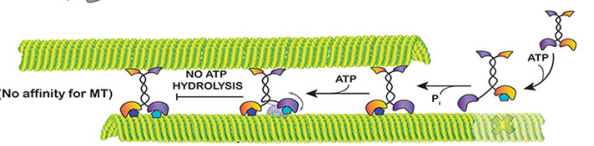

HSET Motor-Stalk (Tailless mutant)

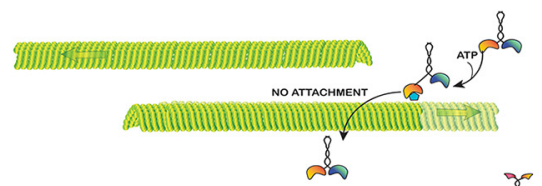

HSET T417N-Rigor (Rigor Mutant)

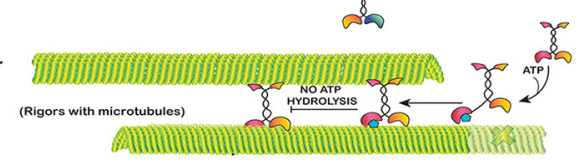

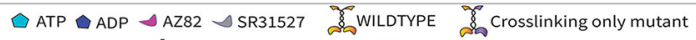
\&ै Tailless mutant

Figure 9. KIFC1 opposes sliding of long microtubules in the axon. $A$, Representative fluorescence images of tubulin signal in non-photoconverted region at 0 and 10 min after photoconversion of Td-Eos tubulin in control and KIFC1-depleted neurons. B, Graph representing percentage of fluorescence decay over 101 min intervals after photoconversion of Td-Eos tubulin in control, KIFC1-depleted, and KIFC1 siRNA + FCPT-, KIFC1 siRNA + 50 nm vinblastine-, KIFC1 siRNA + N593K-, KIFC1 siRNA + HSET-Motor-StalK-,, or KIFC1 siRNA + HSET-Rigor-expressing neurons. * $p<0.05$ for the comparison between control siRNA versus KIFC1 siRNA, ${ }^{\circ} p<0.05$ comparison between KIFC1 siRNA versus KIFC1 siRNA +FCPT, $\wedge p<0.05$ comparison between KIFC1 siRNA versus KIFC1 siRNA $+50 \mathrm{~nm}$ vinblastine, $\sim p<0.05$ comparison between $\mathrm{KIFC1}$ siRNA + HSET N593K, ${ }^{+} p<0.05$ comparison between KIFC1 1 siRNA versus $\mathrm{KIFC} 1$ siRNA + HSET Rigor; $n=10$ per group, except $\mathrm{KIFC1} \mathrm{siRNA} n=12$. C, Graph representing the average fluorescence intensity plot of tubulin along the length of the distal axon from control siRNA, KIFC1-depleted neurons and KIFC1 siRNA + FCPT-, KIFC1 siRNA + 50 nm vinblastine-, KIFC1 siRNA + N593K-, KIFC1 siRNA + HSET-Motor-Stalk-, and KIFC1 siRNA + HSET-Rigor-expressing neurons. * $p<0.05$ for the comparison between control siRNA versus KIFC1 siRNA, ${ }^{\circ} p<0.05$ for the comparison between $\mathrm{KIFC1}$ siRNA versus KIFC1 siRNA+FCPT, $\wedge p<0.05$ for the comparison between KIFC1 siRNA (Figure legend continues.) 
with the idea that there would be greater decay of photoconverted signal with more microtubules sliding into the nonphotoconverted regions. Consistent with this prediction, the percentage of decay of the fluorescence signal after $10 \mathrm{~min}$ in KIFC1-depleted neurons was notably higher than in control neurons (Fig. 9A-D, Movies 5 and 6). To further validate that this decay in fluorescence signal was due to increased microtubule sliding rather than the increased microtubule dynamics suggested by the faster rates of EB3 comets, we measured the appearance of red signal after $10 \mathrm{~min}$ in the non-photoconverted region of the axon as an indication of the sliding of microtubules into that region from the converted region. Plotting the average intensity peaks of the distal axon alone indicted that the levels of converted red fluorescence in the non-photoconverted regions of the axon after 10 min were significantly higher in KIFC1-depleted neurons than in control neurons (Fig. 9C). The peak of the fluorescence intensity curve shifted in KIFC1-depleted neurons compared with control neurons, which indicates the sliding of the photoconverted region (Fig. 9C,D, Movies 5 and 6). We inferred this by measuring the width of the peak using the FWHM function of the software and observing the direction of fluorescence movement in the kymograph, which indicated the distribution of the red fluorescence signal was distributed more along the length of the axon (Fig. 9E, F, respectively).

KIFC1's ability to cross-link microtubules depends on two microtubule-interacting sites, one within the motor domain and the other within the tail domain. Because both microtubuleinteracting sites contribute to restricting microtubule sliding (Braun et al., 2017), truncating either the motor domain or the tail domain such that its microtubule-interacting site is eliminated will lead to an impairment in KIFC1's ability to act as a cross-linker. To further confirm the need for KIFC1's crosslinking properties to explain our results, we used a tail-less mutant called HSET-motor-stalk KIFC1 that consists of 145673 aa. With this mutant expressed in KIFC1-depleted neurons rather than the cross-linking-only mutant, we saw higher decay of signal in the photoconverted region and increase in signal in the non-photoconverted zones, indicating sliding of microtubules from the photoconverted zone into the non-

$\leftarrow$

(Figure legend continued.) versus KIFC1 siRNA $+50 \mathrm{~nm}$ vinblastine, $\sim p<0.05$ for the comparison between $\mathrm{KIFC} 1$ siRNA + HSET N593K, ${ }^{+} p<0.05$ for the comparison between $\mathrm{KIFC} 1$ siRNA versus KIFC1 1 siRNA + HSET Rigor; $n=10$ per group, except KIFC1 siRNA, where $n=12$. D, Representative fluorescence images of the distal axon where Td-Eos was photoconverted. Images represented from 0 and 10 min displaying the decay in fluorescence signal of control and depletion groups. $\boldsymbol{E}$, Bar graph representing the width of the peak tubulin fluorescence intensity after photoconversion of fluorescence signal moving into the non-photoconverted zone. ${ }^{*} p<$ 0.05. Control siRNA, $n=10$; KIFC1 siRNA, $n=12$. $\boldsymbol{F}$, Representative immunofluorescence images and kymograph represent tubulin signal shift in $10 \mathrm{~min}$. G, Schematic illustration of the mechanisms of action of KIFC1 drugs and KIFC1 mutants. Full-length wild-type KIFC1 walks toward the minus-end of one microtubule while binding to another in an ATP-independent fashion, thus resulting in sliding of microtubules relative to one another. Both small-molecule inhibitors (AZ82 and SR31527) of KIFC1 block the ATP activity in the motor domain of KIFC1, which results in KIFC1 dissociation from the microtubules. A cross-linking-only mutant (HSETN593K), which uncouples microtubule binding and microtubule-stimulated ATPase activity, has the ability to cross-link microtubules but cannot slide/transport them. KIFC1-truncated mutant (termed HSET-Motor-Stalk, consisting of 145-673 aa) does not contain the tail domain and thus cannot slide or cross-link microtubules. The KIFC1 rigor mutant (HSET-Rigor-T417N) binds to microtubules and locks them in place, thus preventing microtubule sliding. Data are represented as mean \pm SEM. Unless specified, all statistical test comparisons were done against the control siRNA group with KIFC1 siRNA and/or KIFC1 siRNA+FCPT or KIFC1 siRNA + 50 nM vinblastine or KIFC1 siRNA + N593K or KIFC1 siRNA + HSET-Motor-Stalk and KIFC1 siRNA+HSET-Rigor group using ANOVA test for multiple comparisons. Scale bars: $A, 50$ $\mu \mathrm{m} ; \boldsymbol{D}$ and $\boldsymbol{F}, 10 \mu \mathrm{m}$

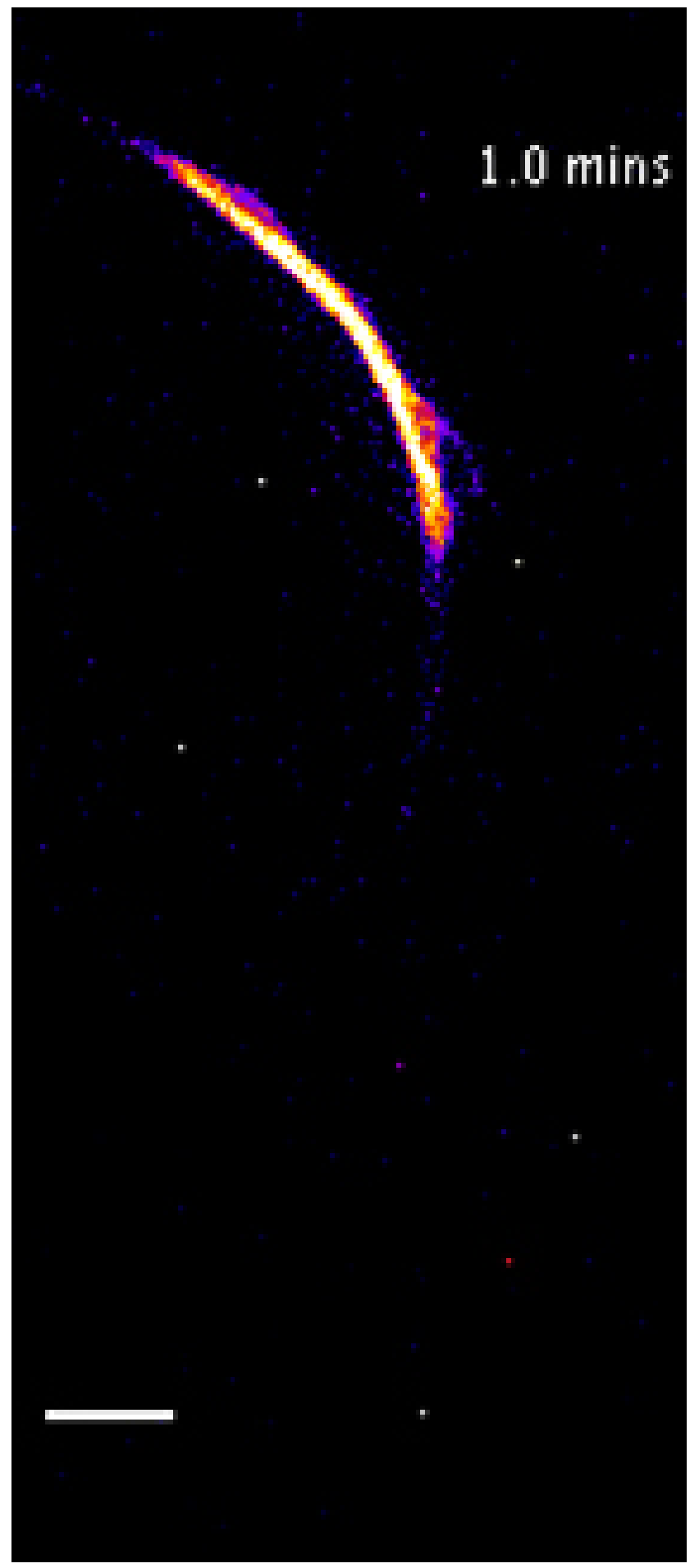

Movie 5. Microtubule sliding in control siRNA-treated neurons. Movie is related to Figure 9 and shows pseudocolored microtubule sliding with Td-Eos tubulin and treated with control siRNA. Scale bar, $10 \mu \mathrm{m}$.

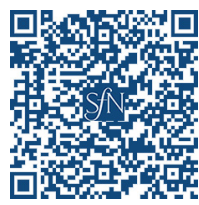

photoconverted zones. Therefore, there was no rescue of the depletion phenotype without the expressed protein being able to cross-link microtubules.

To further test our interpretation that these effects are indicative of microtubule sliding and not microtubule dynamics, we 


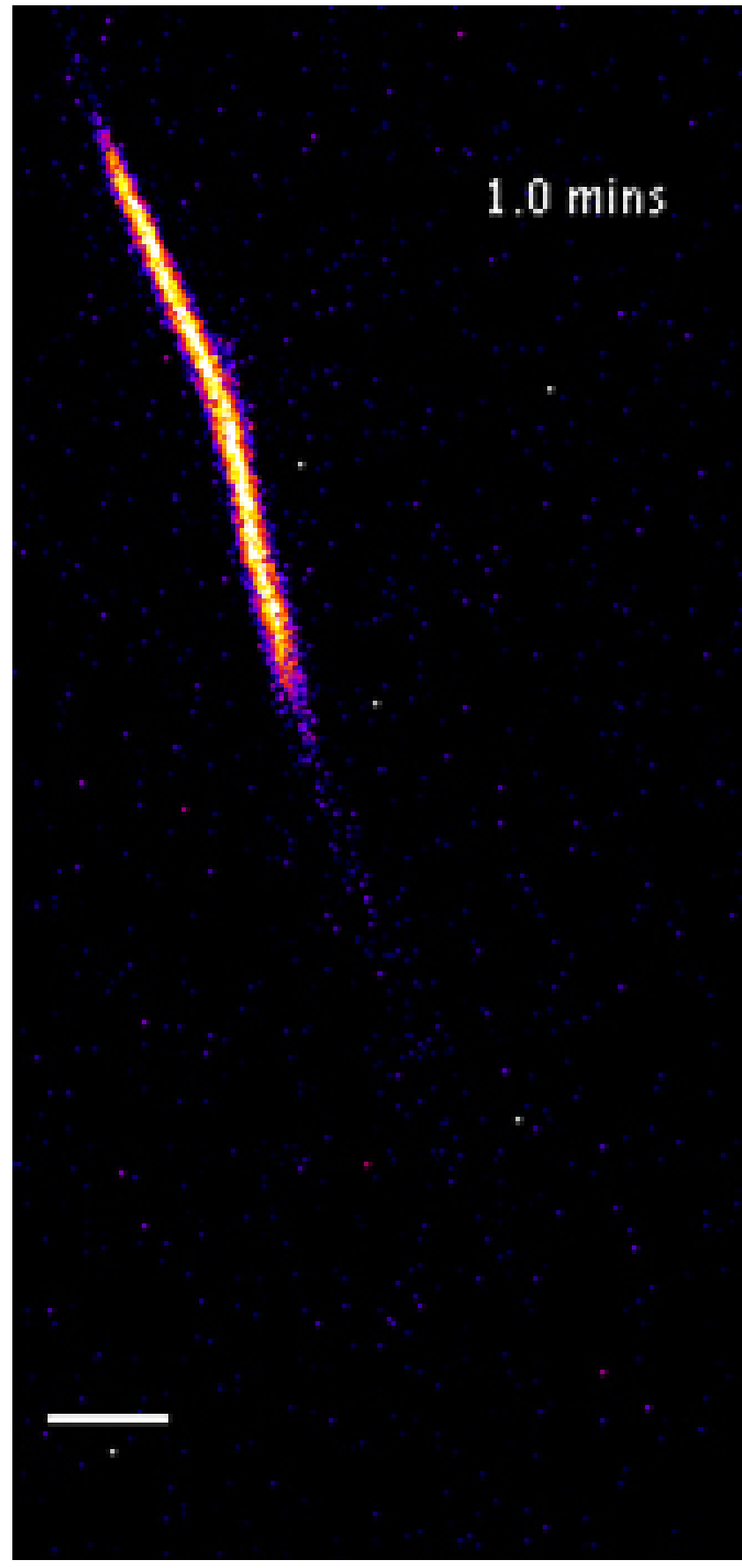

Movie 6. Microtubule sliding in KIFC1 siRNA-treated neurons. Movie is related to Figure 9 and shows pseudocolored microtubule sliding with Td-Eos tubulin and treated with KIFC1 siRNA. Scale bar, $10 \mu \mathrm{m})$.

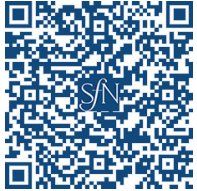

repeated the experiments using drugs that specifically suppress either microtubule dynamics or microtubule transport. When used at $50 \mathrm{~nm}$, vinblastine accomplishes the former (Ahmad et al., 1998), whereas FCPT at $50 \mu \mathrm{M}$ accomplishes the latter (Rao et al., 2016; Rao and Baas, 2018). We found that vinblastine did not alter the observed redistribution of fluorescence, but FCPT entirely prevented it. Finally, we generated a rigor mutant of KIFC1 (T417N, referred to here as KIFC1 rigor mutant), in which a single amino acid mutation causes KIFC1 to tightly bind to mi- crotubules regardless of its nucleotide state, thus forming a tightly bound complex with microtubules that locks them in place and prevents microtubule sliding. We found that expression of this mutant produced the same result as the FCPT. These results are consistent with our interpretation that the observed redistribution of fluorescence in these experiments is due to microtubule sliding and not microtubule dynamics.

\section{Discussion}

A great deal of previous work on KIFC1 in non-neuronal contexts indicates that it can slide apart oppositely oriented microtubules and slide together commonly-oriented microtubules by drawing microtubules together as it slides toward their minus ends (She and Yang, 2017). In addition, KIFC1 can cross-link microtubules via its ATP-independent microtubule-interacting sites (one in the tail domain and one in the motor domain) and thereby prevent the microtubules from moving apart via forces generated by other motor proteins (Cai et al., 2009; She and Yang, 2017). In testing for such a mechanism of KIFC1 in neurons, we used siRNA-based depletion of KIFC1, two different KIFC1 drugs and three different KIFC1 mutant constructs, as well as the wild-type KIFC1 construct (Fig. 7E). The two drugs, AZ82 and SR31527, were both demonstrated to be effective and specific in neurons by the fact that their effects phenocopied the siRNA in several different ways and by the fact that the drugs had no demonstrable effects on axons that had been depleted of KIFC1 by siRNA. In addition, the two drugs elicited the same effects on the neurons despite having somewhat different mechanisms of action, with AZ82 binding to KIFC1 only if it is already bound to a microtubule and SR31527 binding to KIFC1 even when not bound to a microtubule (Wu et al., 2013; Yang et al., 2014; Zhang et al., 2016; Park et al., 2017). Therefore, AZ82 dissociates KIFC1 from the microtubule, whereas SR31527 prevents its association with the microtubule. KIFC1 requires ATPase activity to interact with microtubules, so that even the ATP-independent cross-linking properties of KIFC1 are curtailed by the drugs. The cross-linkingonly mutant produces a different result from the drugs because it is able to dimerize with wild-type KIFC1 that remains after the protein is partially depleted by the siRNA and thus can interact with the microtubule via the wild-type protein but cannot slide microtubules due to the mutant protein. The HSET-motor-stalk mutant lacks the tail domain of KIFC1 and thus lacks one of the microtubule-interacting sites required for KIFC1's microtubulecross-linking ability. The rigor-mutant acts like the crosslinking-only-mutant except that, once the microtubules are cross-linked, they are held irreversibly in place.

Our initial hypothesis about KIFC1 in neurons was that it acts as a backup to cytoplasmic dynein's role in transporting and organizing microtubules (Sharp et al., 1997; Baas et al., 2006; Rao and Baas, 2018). Some aspects of the depletion phenotype are reminiscent of what has been observed in the past with inhibition of cytoplasmic dynein or its partner proteins. Specifically, axons are shorter and more prone to retraction and microtubules fail to invade the lamellar regions of growth cones and filopodia. However, despite similarities to dynein-based phenotypes, the mechanistic explanation for these phenomena appears to be quite different in the case of KIFC1. Both dynein and KIFC1 are relevant to opposing myosin-based forces that promote axonal retraction via contractility of cortical actin as well as retrograde flow of the actin cytoskeleton in the growth cone. In the case of dynein-compromised neurons, microtubules struggle against the retrograde actin flow and fail to invade the lamellar region of the growth cone and, in the case of KIFC1-compromised neurons, 
the same is true. However, in the case of KIFC1-compromised neurons, the microtubules have a greater propensity to curl, sometimes making a full U-turn, which may account for the greater propensity for microtubule polarity flaws in the distal third of the axon compared with the rest of the axon. As a result, microtubules cannot appropriately fan out into their lamellar regions and growth cones take on the morphology and behavior of stalled growth cones. In the case of dynein-compromised neurons, axons retract more readily and to a greater extent, but display the same sinusoidal bending along their lengths as control axons when they retract. By contrast, axons of KIFC1-compromised neurons also retract more readily than control axons, but fail to show the sinusoidal bending. Given that the sinusoidal bending is due to long microtubules that resist backward sliding (Shaw and Bray, 1977; Baas and Heidemann, 1986; Baas et al., 1987; George et al., 1988; He et al., 2002), these results indicate that microtubules slide backward with less resistance in the absence of KIFC1 than in the absence of dynein.

Curiously, in none of our experiments, not even the short microtubule transport assay, did we observe a net decrease in microtubule sliding when KIFC1 was depleted or inhibited. Instead, we observed mal-oriented and misaligned microtubules, a change in the ratio of short microtubules moving in each direction in the axon, and a diminution in the capacity of the microtubule array to resist forces that would otherwise slide them. The mal-orientation and misalignment of microtubules in KIFC1compromised axons are presumably due to both lack of appropriate sliding of microtubules by KIFC1 as well as abnormal sliding of microtubules by other motor proteins. The retrograde transport of short microtubules in the axon is believed to be a mechanism for clearing the axon of minus-end-out microtubules (Zheng et al., 2008; Baas and Mozgova, 2012; Rao and Baas, 2018), but in the absence of KIFC1, the greater anterograde transport of short microtubules together with flaws in microtubule polarity orientation suggest that this polarity-sorting mechanism has gone awry. Computational modeling indicates that cross-linker proteins are needed to limit the transport of microtubules to the specific motor protein, namely cytoplasmic dynein, that is able to appropriately polarity sort the microtubules (Rao and Baas, 2018). The present results are consistent with KIFC1 playing such a role, with the depletion of KIFC1 resulting in microtubule polarity flaws along the length of the axon (not just in its distal third, which has heightened polarity flaws, presumably due to the curling back of microtubule from the growth cone). Therefore, KIFC1 joins a growing list of proteins relevant to preserving the microtubule polarity pattern of the axon (Ehaideb et al., 2014; van Beuningen et al., 2015; Rao et al., 2017; Kelliher et al., 2018).

The studies with the KIFC1 mutants demonstrate (Fig. 9G) that KIFC1 is not just a cross-linker, nor can the panoply of its depletion or inhibition effects be explained exclusively by its cross-linking properties. For example, the cross-linking-only mutant was able to partially but not completely rescue aspects of the KIFC1 depletion phenotype, indicating that KIFC1's energyburning properties are also crucial to its mechanism of action. Axons remain short when KIFC1 can cross-link microtubules but not burn energy and the microtubules are spaced farther apart than they should be. We conclude that, in the axon, KIFC1 binds to microtubules and slides them into alignment in an ATPdependent fashion and then cross-links them in an ATPindependent fashion to oppose their subsequent sliding by other motors. We also found that KIFC1 depletion results in a modest increase in microtubule dynamics, but the KIFC1 phenotypes appear to be best explained by a defective microtubule sliding/ cross-linking mechanism.

For decades, KIFC1 was presumed to be absent from neurons, showing up in brain libraries only because of non-neuronal mitotic cells in the tissue (Saito et al., 1997). Now, it appears that KIFC1 may provide answers to several unanswered questions in neuronal cell biology. For example, growth cone stalling has long been observed but with no clear mechanistic explanation (Szebenyi et al., 1998; Dent et al., 1999). When a growth cone is in a stalling mode, KIFC1 may be disengaged from the microtubules and then, when the stalling episode is complete, it may once again engage the microtubules. Similarly, the precise characteristics of axonal retraction may be determined by the levels of KIFC1 associated with the microtubules. Such events may be under the control of environmental factors that elicit signaling cascades relevant to KIFC1. KIFC1 can be degraded by a CDK1-dependent event (Singh et al., 2014) or another phosphorylation pathway (Molodtsov et al., 2016; Beaven et al., 2017), either of which could thrust a motile growth cone into a period of stalling or an axon into a bout of retraction. Dendrites might use KIFC1 quite differently from axons given that dendrites contain microtubules of mixed polarity orientation (Baas et al., 1991; Sharp et al., 1995; Lin et al., 2012; Yau et al., 2016). In theory, KIFC1 could be the unknown factor that cross-links dendritic microtubules into place once their mixed orientation has been achieved (Lin et al., 2012) or it could work in a regulated fashion to keep the proportion of microtubules of each orientation optimized through spurts of anti-parallel microtubule sliding. Finally, the fact that KIFC1 continues to be expressed in adult neurons potentially implicates KIFC1 as a factor in preventing axons from retracting back from the synapses, which we posit could provide a means for fortification of axons against die-back during neurodegenerative diseases.

\section{References}

Ahmad FJ, Echeverri CJ, Vallee RB, Baas PW (1998) Cytoplasmic dynein and dynactin are required for the transport of microtubules into the axon. J Cell Biol 140:391-401.

Ahmad FJ, Hughey J, Wittmann T, Hyman A, Greaser M, Baas PW (2000) Motor proteins regulate force interactions between microtubules and microfilaments in the axon. Nat Cell Biol 2:276-280.

Ahmad FJ, He Y, Myers KA, Hasaka TP, Francis F, Black MM, Baas PW (2006) Effects of dynactin disruption and dynein depletion on axonal microtubules. Traffic 7:524-537.

Applegate KT, Besson S, Matov A, Bagonis MH, Jaqaman K, Danuser G (2011) plusTipTracker: quantitative image analysis software for the measurement of microtubule dynamics. J Struct Biol 176:168-184.

Baas PW (2002) Microtubule transport in the axon. Int Rev Cytol 212:4162.

Baas PW, Ahmad FJ (2001) Force generation by cytoskeletal motor proteins as a regulator of axonal elongation and retraction. Trends Cell Biol 11: 244-249.

Baas PW, Heidemann SR (1986) Microtubule reassembly from nucleating fragments during the regrowth of amputated neurites. J Cell Biol 103: 917-927.

Baas PW, Mozgova OI (2012) A novel role for retrograde transport of microtubules in the axon. Cytoskeleton (Hoboken) 69:416-425.

Baas PW, White LA, Heidemann SR (1987) Microtubule polarity reversal accompanies regrowth of amputated neurites. Proc Natl Acad Sci U S A 84:5272-5276.

Baas PW, Slaughter T, Brown A, Black MM (1991) Microtubule dynamics in axons and dendrites. J Neurosci Res 30:134-153.

Baas PW, Vidya Nadar C, Myers KA (2006) Axonal transport of microtubules: the long and short of it. Traffic 7:490-498.

Banker G (2018) The development of neuronal polarity: a retrospective view. J Neurosci 38:1867-1873.

Beaven R, Bastos RN, Spanos C, Romé P, Cullen CF, Rappsilber J, Giet R, 
Goshima G, Ohkura H (2017) 14-3-3 regulation of ncd reveals a new mechanism for targeting proteins to the spindle in oocytes. J Cell Biol 216:3029-3039.

Braun M, Drummond DR, Cross RA, McAinsh AD (2009) The kinesin-14 Klp2 organizes microtubules into parallel bundles by an ATP-dependent sorting mechanism. Nat Cell Biol 11:724-730.

Braun M, Lansky Z, Szuba A, Schwarz FW, Mitra A, Gao M, Lüdecke A, Ten Wolde PR, Diez S (2017) Changes in microtubule overlap length regulate kinesin-14-driven microtubule sliding. Nat Chem Biol 13:1245-1252.

Buster DW, Baird DH, Yu W, Solowska JM, Chauvière M, Mazurek A, Kress M, Baas PW (2003) Expression of the mitotic kinesin Kif15 in postmitotic neurons: implications for neuronal migration and development. J Neurocytol 32:79-96.

Cai S, Weaver LN, Ems-McClung SC, Walczak CE (2009) Kinesin-14 family proteins HSET/XCTK2 control spindle length by cross-linking and sliding microtubules. Mol Biol Cell 20:1348-1359.

Del Castillo U, Lu W, Winding M, Lakonishok M, Gelfand VI (2015) Pavarotti/MKLP1 regulates microtubule sliding and neurite outgrowth in drosophila neurons. Curr Biol 25:200-205.

Dent EW, Callaway JL, Szebenyi G, Baas PW, Kalil K (1999) Reorganization and movement of microtubules in axonal growth cones and developing interstitial branches. J Neurosci 19:8894-8908.

Ehaideb SN, Iyengar A, Ueda A, Iacobucci GJ, Cranston C, Bassuk AG, Gubb D, Axelrod JD, Gunawardena S, Wu CF, Manak JR (2014) prickle modulates microtubule polarity and axonal transport to ameliorate seizures in flies. Proc Natl Acad Sci U S A 111:11187-11192.

Falnikar A, Tole S, Baas PW (2011) Kinesin-5, a mitotic microtubuleassociated motor protein, modulates neuronal migration. Mol Biol Cell 22:1561-1574.

Ferhat L, Kuriyama R, Lyons GE, Micales B, Baas PW (1998a) Expression of the mitotic motor protein CHO1/MKLP1 in postmitotic neurons. Eur J Neurosci 10:1383-1393.

Ferhat L, Cook C, Chauviere M, Harper M, Kress M, Lyons GE, Baas PW (1998b) Expression of the mitotic motor protein Eg5 in postmitotic neurons: implications for neuronal development. J Neurosci 18:7822-7835.

Fink G, Hajdo L, Skowronek KJ, Reuther C, Kasprzak AA, Diez S (2009) The mitotic kinesin-14 ncd drives directional microtubule-microtubule sliding. Nat Cell Biol 11:717-723.

George EB, Schneider BF, Lasek RJ, Katz MJ (1988) Axonal shortening and the mechanisms of axonal motility. Cell Motil Cytoskeleton 9:48-59.

Grabham PW, Seale GE, Bennecib M, Goldberg DJ, Vallee RB (2007) Cytoplasmic dynein and LIS1 are required for microtubule advance during growth cone remodeling and fast axonal outgrowth. J Neurosci 27:5823-5834.

Hanlon DW, Yang Z, Goldstein LS (1997) Characterization of KIFC2, a neuronal kinesin superfamily member in mouse. Neuron 18:439-451.

He Y, Yu W, Baas PW (2002) Microtubule reconfiguration during axonal retraction induced by nitric oxide. J Neurosci 22:5982-5991.

He Y, Francis F, Myers KA, Yu W, Black MM, Baas PW (2005) Role of cytoplasmic dynein in the axonal transport of microtubules and neurofilaments. J Cell Biol 168:697-703.

Hepperla AJ, Willey PT, Coombes CE, Schuster BM, Gerami-Nejad M, McClellan M, Mukherjee S, Fox J, Winey M, Odde DJ, O’Toole E, Gardner MK (2014) Minus-end-directed kinesin-14 motors align antiparallel microtubules to control metaphase spindle length. Dev Cell 31:61-72.

Kelliher MT, Yue Y, Ng A, Kamiyama D, Huang B, Verhey KJ, Wildonger J (2018) Autoinhibition of kinesin-1 is essential to the dendrite-specific localization of Golgi outposts. J Cell Biol 217:2531-2547.

Lin S, Liu M, Son YJ, Timothy Himes B, Snow DM, Yu W, Baas PW (2011) Inhibition of kinesin-5, a microtubule-based motor protein, as a strategy for enhancing regeneration of adult axons. Traffic 12:269-286.

Lin S, Liu M, Mozgova OI, Yu W, Baas PW (2012) Mitotic motors coregulate microtubule patterns in axons and dendrites. J Neurosci 32:1403314049.

Liu M, Nadar VC, Kozielski F, Kozlowska M, Yu W, Baas PW (2010) Kinesin-12, a mitotic microtubule-associated motor protein, impacts axonal growth, navigation, and branching. J Neurosci 30:14896-14906.

Molodtsov MI, Mieck C, Dobbelaere J, Dammermann A, Westermann S, Vaziri A (2016) A Force-Induced Directional Switch of a Molecular Motor Enables Parallel Microtubule Bundle Formation. Cell 167:539-552.e14.

Mountain V, Simerly C, Howard L, Ando A, Schatten G, Compton DA
(1999) The kinesin-related protein, HSET, opposes the activity of Eg5 and cross-links microtubules in the mammalian mitotic spindle. J Cell Biol 147:351-366.

Myers KA, Baas PW (2007) Kinesin-5 regulates the growth of the axon by acting as a brake on its microtubule array. J Cell Biol 178:1081-1091.

Myers KA, Tint I, Nadar CV, He Y, Black MM, Baas PW (2006) Antagonistic forces generated by cytoplasmic dynein and myosin-II during growth cone turning and axonal retraction. Traffic 7:1333-1351.

Nadar VC, Ketschek A, Myers KA, Gallo G, Baas PW (2008) Kinesin-5 is essential for growth-cone turning. Curr Biol 18:1972-1977.

Nadar VC, Lin S, Baas PW (2012) Microtubule redistribution in growth cones elicited by focal inactivation of kinesin-5. J Neurosci 32:5783-5794.

Oladipo A, Cowan A, Rodionov V (2007) Microtubule motor ncd induces sliding of microtubules in vivo. Mol Biol Cell 18:3601-3606.

Park HW, Ma Z, Zhu H, Jiang S, Robinson RC, Endow SA (2017) Structural basis of small molecule ATPase inhibition of a human mitotic kinesin motor protein. Sci Rep 7:15121.

Qiang L, Yu W, Andreadis A, Luo M, Baas PW (2006) Tau protects microtubules in the axon from severing by katanin. J Neurosci 26:3120-3129.

Rao AN, Baas PW (2018) Polarity sorting of microtubules in the axon. Trends Neurosci 41:77-88.

Rao AN, Falnikar A, O'Toole ET, Morphew MK, Hoenger A, Davidson MW, Yuan X, Baas PW (2016) Sliding of centrosome-unattached microtubules defines key features of neuronal phenotype. J Cell Biol 213:329-341.

Rao AN, Patil A, Black MM, Craig EM, Myers KA, Yeung HT, Baas PW (2017) Cytoplasmic dynein transports axonal microtubules in a polaritysorting manner. Cell Rep 19:2210-2219.

Saito N, Okada Y, Noda Y, Kinoshita Y, Kondo S, Hirokawa N (1997) KIFC2 is a novel neuron-specific C-terminal type kinesin superfamily motor for dendritic transport of multivesicular body-like organelles. Neuron 18:425-438.

Sharp DJ, Yu W, Baas PW (1995) Transport of dendritic microtubules establishes their nonuniform polarity orientation. J Cell Biol 130:93103

Sharp DJ, Kuriyama R, Essner R, Baas PW (1997) Expression of a minusend-directed motor protein induces Sf9 cells to form axon-like processes with uniform microtubule polarity orientation. J Cell Sci 110: 2373-2380.

Sharp DJ, Brown HM, Kwon M, Rogers GC, Holland G, Scholey JM (2000) Functional coordination of three mitotic motors in Drosophila embryos. Mol Biol Cell 11:241-253.

Shaw G, Bray D (1977) Movement and extension of isolated growth cones. Exp Cell Res 104:55-62.

She ZY, Yang WX (2017) Molecular mechanisms of kinesin-14 motors in spindle assembly and chromosome segregation. J Cell Sci 130:2097-2110.

Silverman MA, Kaech S, Ramser EM, Lu X, Lasarev MR, Nagalla S, Banker G (2010) Expression of kinesin superfamily genes in cultured hippocampal neurons. Cytoskeleton (Hoboken) 67:784-795.

Simeonov DR, Kenny K, Seo L, Moyer A, Allen J, Paluh JL (2009) Distinct kinesin-14 mitotic mechanisms in spindle bipolarity. Cell Cycle 8:35713583.

Singh SA, Winter D, Kirchner M, Chauhan R, Ahmed S, Ozlu N, Tzur A, Steen JA, Steen H (2014) Co-regulation proteomics reveals substrates and mechanisms of APC/C-dependent degradation. EMBO J 33:385-399.

Song H, Endow SA (1996) Binding sites on microtubules of kinesin motors of the same or opposite polarity. Biochemistry 35:11203-11209.

Stout A, D'Amico S, Enzenbacher T, Ebbert P, Lowery LA (2014) Using plusTipTracker software to measure microtubule dynamics in Xenopus laevis growth cones. J Vis Exp 91:e52138.

Szebenyi G, Callaway JL, Dent EW, Kalil K (1998) Interstitial branches develop from active regions of the axon demarcated by the primary growth cone during pausing behaviors. J Neurosci 18:7930-7940.

van Beuningen SFB, Will L, Harterink M, Chazeau A, van Battum EY, Frias CP, Franker MAM, Katrukha EA, Stucchi R, Vocking K, Antunes AT, Slenders L, Doulkeridou S, Sillevis Smitt P, Altelaar AFM, Post JA, Akhmanova A, Pasterkamp RJ, Kapitein LC, de Graaff E, et al. (2015) TRIM46 Controls Neuronal Polarity and Axon Specification by Driving the Formation of Parallel Microtubule Arrays. Neuron 88:1208-1226.

Walczak CE, Vernos I, Mitchison TJ, Karsenti E, Heald R (1998) A model for the proposed roles of different microtubule-based motor proteins in establishing spindle bipolarity. Curr Biol 8:903-913.

Weaver LN, Ems-McClung SC, Chen SH, Yang G, Shaw SL, Walczak CE 
(2015) The ran-GTP gradient spatially regulates XCTK2 in the spindle. Curr Biol 25:1509-1514.

Wu J, Mikule K, Wang W, Su N, Petteruti P, Gharahdaghi F, Code E, Zhu X, Jacques K, Lai Z, Yang B, Lamb ML, Chuaqui C, Keen N, Chen H (2013) Discovery and mechanistic study of a small molecule inhibitor for motor protein KIFC1. ACS Chem Biol 8:2201-2208.

Yang B, Lamb ML, Zhang T, Hennessy EJ, Grewal G, Sha L, Zambrowski M, Block MH, Dowling JE, Su N, Wu J, Deegan T, Mikule K, Wang W, Kaspera R, Chuaqui C, Chen H (2014) Discovery of potent KIFC1 inhibitors using a method of integrated high-throughput synthesis and screening. J Med Chem 57:9958-9970.

Yang Z, Roberts EA, Goldstein LS (2001) Functional analysis of mouse C-terminal kinesin motor KifC2. Mol Cell Biol 21:2463-2466.
Yau KW, Schätzle P, Tortosa E, Pagès S, Holtmaat A, Kapitein LC, Hoogenraad CC (2016) Dendrites in vitro and in vivo contain microtubules of opposite polarity and axon formation correlates with uniform plus-end-out microtubule orientation. J Neurosci 36:10711085.

Zhang W, Zhai L, Wang Y, Boohaker RJ, Lu W, Gupta VV, Padmalayam I, Bostwick RJ, White EL, Ross LJ, Maddry J, Ananthan S, Augelli-Szafran CE, Suto MJ, Xu B, Li R, Li Y (2016) Discovery of a novel inhibitor of kinesin-like protein KIFC1. Biochem J 473:1027-1035.

Zheng Y, Wildonger J, Ye B, Zhang Y, Kita A, Younger SH, Zimmerman S, Jan LY, Jan YN (2008) Dynein is required for polarized dendritic transport and uniform microtubule orientation in axons. Nat Cell Biol 10:11721180 . 